\title{
IMPROVING LEGIBILITY OF NATURAL DEDUCTION PROOFS IS NOT TRIVIAL
}

\author{
KAROL PĄK \\ e-mail address: pakkarol@uwb.edu.pl \\ University of Bialystok, Institute of Computer Science, Bialystok, Poland
}

\begin{abstract}
In formal proof checking environments such as Mizar it is not merely the validity of mathematical formulas that is evaluated in the process of adoption to the body of accepted formalizations, but also the readability of the proofs that witness validity. As in case of computer programs, such proof scripts may sometimes be more and sometimes be less readable. To better understand the notion of readability of formal proofs, and to assess and improve their readability, we propose in this paper a method of improving proof readability based on Behaghel's First Law of sentence structure. Our method maximizes the number of local references to the directly preceding statement in a proof linearisation. It is shown that our optimization method is NP-complete.
\end{abstract}

\section{INTRODUCTION}

1.1. Motivations. The readability of formal proofs has significantly influenced development of formalization. The idea of formalization was recognized by David Hilbert in his program of formalization of mathematics - research in his program led, in particular, to the concept of the universal Turing machine. Hilbert's project in his most idealistic form collapsed due to Gödel's incompleteness theorems. In spite of them, a group of mathematicians under a single pen name Nicolas Bourbaki tried to formalize an exposition of modern advanced mathematics using first-order logic. This project was also abandoned because the resulting formal proofs turned out to be too obscure and the formalization in such a detailed form started to be perceived as hardly readable [34] and as a result impractical. Moreover, checking the correctness of formal proofs turned out to be as difficult as the reading comprehension of these proofs. Not only Bourbaki's project was faced with the readability problems. The earlier efforts of Whitehead and Russell in the formalization of mathematics had the same problems. The formal definitions and proofs that they created were deemed cryptic as the approach to mathematics they used was extremely rigorous and precise. The formalization

2012 ACM CCS: [Computing methodologies]: Artificial intelligence-Knowledge representation and reasoning;[Theory of computation]: Formal languages and automata theory; Design and analysis of algorithms - Graph algorithms analysis .

Key words and phrases: Operations on languages, Legibility of proofs, Acyclic partition.

The paper has been financed by the resources of the Polish National Science Centre granted by decision no DEC-2012/07/N/ST6/02147.

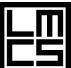

DOI:10.2168/LMCS-10(3:23)2014

(C) Karol Pąk

(c) Creative Commons 
has become feasible only after increasing availability of computers. These problems are described in more detail by Zammit [44].

Proof development in formalized mathematical frameworks is similar to program development. In both cases the content is created in an artificial language that has precise meaning executed by a computer system. Therefore it comes as no surprise that both activities have much in common. Since readability of programs has a significant impact on their maintainability [1] it is strongly expected that readability of proof scripts has a significant impact on proof maintainability. Indeed the maintenance tasks take place in current formal proof development, e.g. novice users of the formal proof development tools often follow the existing formalizations, and the formalizations are subject to refinement for instance when a generalized version of a theorem is to be proved. This concerns especially systems such as Isabelle/Isar [42] or Mizar [28] where the proof script language is close to the natural language.

In this paper, we focus on another, still underdeveloped approach. Models of cognitive perception of read material stress that locality of reference is a significant factor in the process of understanding. This can be summarized with the Behaghel's First Law that can be stated as follows, elements that belong close together intellectually will also be placed close together 3]. This law of local reference is also recognized in modern scientific literature concerning human perception [11, 25]. The significance of the locality of reference is exploited in the current study. We focus on a particular proof step in a proof script. The step uses some information that was derived before in the proof. This information can be located somewhere far away in the proof or within a close neighborhood of the step. With Behaghel's law in mind, we assume that a step where at least part of information it requires is available in the directly preceding step is more comprehensive than a step in which all information is far away in the proof (as a significant part of assumptions can still be in the, so called, working memory, see e.g. [8, Chapter 1]).

The locality of reference principle is the basis for a method of proof distillation that is presented in this paper. Since the steps that refer to the preceding step are perceived as more comprehensive, the procedure transforms the proof scripts so that the number of steps that violate the requirement is minimal. The transformation consists of two actions. The first one translates a linear representation of a proof script to a representation in the form of a directed acyclic graph and the second one chooses a different linearisation of the proof so that the number of proof steps that are, according to the presented principles, difficult to comprehend is minimal.

1.2. Related works. The experience of big proof formalization developments shows that formalized results are often used as libraries, which requires reading proof scripts [16]. But there are many more reasons that force formalization authors to read existing proof scripts. New users often use the available library as an in-depth tutorial [29. Experienced users adapt or modify existing proofs to obtain stronger theorems [14] (actually this is mentioned in page 3 of an unpublished preliminary version of the article [13]).

Authors that cooperate on a common formalization analyze each other's achievements (e.g. over $40 \%$ of MML proof scripts have more that one author). Therefore it comes as no surprise that formal systems develop in many directions that improve their readability.

One of the most popular methods to make it possible for computers to assist in formal reasoning is based on bringing the formal mathematical language to the informal one by introduction to the formal language idioms that stem from informal mathematical practice. 
In particular, the keywords thus and show(s) indicate a conclusion of reasoning in Mizar and Isabelle/Isar systems respectively (for more details see [15, 42]). In both systems the same assume(s) keyword is used to indicate a step which, e.g., assumes the antecedent of a thesis that is an implication. Such constructions, which accord to the rules of natural deduction created by S. Jaśkowski and F. B. Fitch [9, 19] were introduced in the Mizar system and later implemented in a number of procedural systems: Declare [35], Mizar Mode for $H O L$ [17, Mizar-light for HOL-light [43, miz3 for HOL-light [2, MMode for Coq [12, declarative proof language (DPL) for Coq [7]. The process of making the Mizar language similar to the informal one is based also on introduction of informal constructions such as ellipsis [22]. In particular, in the MML database we can find theorems formulated as follows: $\mathrm{k}<=\mathrm{n}$ implies $\mathrm{k}=0$ or $\ldots$ or $\mathrm{k}=\mathrm{n}$, and this simply states that for every natural numbers $k$ and $n$, if $k \leq n$, then $k$ can be equal to 1 or 2 or ... or $n$.

Mizar developers are generally very careful when adding new syntactic constructs to the language, and always prefer symbols that stem from standard English. All logical symbols in Mizar, as well as symbols denoting functors, predicates and attributes in the Mizar language are typed as plain text in the editor (the editor of choice for a majority of Mizar users is MizarMode for Emacs created by Urban [39]). Conversion of text symbols to $\mathrm{LT}_{\mathrm{E}} \mathrm{X}$ is performed only when the proof script is published in the dedicated journal "Formalized Mathematics". In this respect, the editor used for Isabelle/Isar-jEdit [41] is more flexible and can visualize proofs both as plain text or in $\mathrm{LT}_{\mathrm{E}} \mathrm{X}$ symbols. Clearly these editors provide many features typical for programming language editors such as syntax highlighting or hint systems (infotip). Additionally, MizarMode is connected with the Automated Reasoning for Mizar (MizAR $)$ service that provides ATP assistance to Mizar authors in searching a proof of a given statement [40] and also facilitates access to the tools distributed with the Mizar system, which can to some extent improve the proof scripts legibility. This initial improvement generally is based on finding and removing irrelevant parts of reasoning or modifying the justification of steps to simplify the list of used premises, preserving the correctness of the modified proof scripts. In particular, the utility, called Irrelevant Premises Detector (distributed under the name RELPREM), indicates premises which are not needed to justify a given step in reasoning (for more details see [15]). Another, equally popular utility, Irrelevant Inferences Detector (RELINFER), is often used by authors when they complete writing a Mizar article. This tool indicates every reference to an irrelevant step, i.e. a reference which can be replaced by all references used to justify this step without affecting the correctness of reasoning. In consequence such modification can shorten proof scripts, but also can spoil their readability. Indeed, steps irrelevant for the machine can be crucial for human understanding of the proof idea. It is common that inferences which are obvious for proof writers are not obvious for the checker and vice versa [32]. Moreover, a simple variant of resolution that is implemented in the Mizar system does not approximate well these two kinds of obviousness. As a result the Mizar developers distribute only tools that enable finding such references, but the decision about their removal is left to the proof author to make it possible to preserve proof legibility.

Independent efforts of Urban concerning visualization of existing Mizar proof scripts led to creation of an auxiliary tool that converts every script to a linked HTML form ([38], see also http://mizar.org/version/current/html/). This linked form includes additional information that is not explicitly available in the script, but is calculated by the Mizar analyzer during the verification process. In particular, it gives immediate access to every definition that has been used implicitly in reasoning and every definition of functor, predicate, and 
attribute that appears in the statement of a proof step. It is worth to note that the MML script authors often use the same symbol to express different things, e.g., * and + are defined or redefined for different notions in MML more than 130 and 160 times, respectively. Therefore, it comes as no surprise that in scripts with a more complex environment, even the authors have a problem in recognizing the symbol variant they used.

Another direction of improving the legibility of Mizar proof scripts is the automation of the most frequently performed operations in reasoning, which was explored by Naumowicz and Byliński [27. This approach is based on the fact that premises that are simply a substitution of some functor (e.g., commutativity, involutivnes) or predicate (e.g., reflexivity, symmetry) do not have to be formulated explicitly in reasoning if these properties have been already proven and substituted terms occur in the reasoning. A special kind of information that is automatically generated and used in justifications of the Mizar proof steps, called reduction, has been implemented by Korniłowicz [23]. Here, a reduction means an equality $f\left(t_{1}, t_{2}, \ldots, t_{n}\right)=t$ where $f$ is an $n$-ary function symbol, $t_{1}, t_{2}, \ldots, t_{n}$ are terms, and $t$ is a subterm of $f\left(t_{1}, t_{2}, \ldots, t_{n}\right)$, e.g., the restriction of a function $f$ to its domain is equal to $f$.

An orthogonal approach to improving the legibility of formal proof scripts is based on popular practices that solve the problem of illegible long reasoning in informal mathematical practice. This approach is generally based on two methods. The first one consists in finding (often less important) fragments of reasoning and then extracting them in the form of a lemma, or encapsulating them at deeper levels of nested proofs. The second ones tries to reorganize the order of independent steps focusing mainly on premises used to justify proof steps. The first experiments with the first method were carried out generally to reduce the size of mechanically generated proofs [31. It is generally known that theorem provers often justify the same goal and in exactly the same way several times. Therefore, it is not surprising that the effort to extract similar proofs as lemmas is often undertaken. However, such modifications are generally carried out without the emphasis on the legibility of obtained scripts. Only the initial studies on properties of reasoning passages that in the reader's opinion determine fragments that deserve to be called a lemma were conducted [30]. Additionally, there were considered dependencies between such properties and the complication level of statements that describe reasoning in selected passages.

Experiential tools that try to realize the second approach were created by Pąk for the Mizar system [29. The impact of these tools, which are based on greedy algorithms, has been already recognized by the community of Mizar authors [30. Additionally, these techniques of proof script modification are taken into consideration in other systems as an effective way to refactor machine proofs [5, 20, 33. However, techniques that try to optimise proof readability criteria in most cases correspond to problems for which complexity is still an open problem.

In this paper we study the computational complexity of methods that have origins in Behaghel's law. For this we devise a generalization of two known NP-complete optimization problems that have been already described in 10 and reduce to these problems two proof readability criteria. This shows that the task of improving readability is computationally demanding and so non-trivial.

In Section 2 we introduce the notion of an abstract model of natural deduction proofs and we discuss selected methods to improve readability. In Section 3 we restrict our attention to two problems of improving the legibility of natural deduction proofs that have origins in Behaghel's law. Then in Section 4 we formalize these problems in terms of the acyclic 
partitions and in Section 5 we show that these problems are NP-complete, since Minimum Feedback Arc Set problem can be transformed to them. In Section 6 we present a family of abstract proof graphs for which there exist proofs written in the Mizar system that have structures described by these graphs. Section 7 contains a brief overview of other problems associated with improving proof legibility and the study of their complexity. Finally, Section 8 concludes the paper and discusses the future work.

\section{Graph Representation of PROOFS}

The abstract notion of proof we deal with here is based on natural deduction created by S. Jaśkowski and F. B. Fitch [9, 19, 26]. As an illustration, let us consider an example written in this notation, which is presented in Fig. 1. In the same way as M. Hurt and M. Ryan [18],

\begin{tabular}{|c|c|c|c|}
\hline 1 & & $\exists_{x} P(x)$ & premise \\
\hline 2 & & $\forall_{x}(P(x) \rightarrow Q(x))$ & premise \\
\hline 3 & & $\forall_{x} \forall_{y}(Q(x) \rightarrow R(y))$ & premise \\
\hline 4 & $y_{0}$ & & \\
\hline 5 & $x_{0}$ & $P\left(x_{0}\right)$ & assumption \\
\hline 6 & & $P\left(x_{0}\right) \rightarrow Q\left(x_{0}\right)$ & $\forall_{x}$ e 2 \\
\hline 7 & & $\forall_{y}\left(Q\left(x_{0}\right) \rightarrow R(y)\right)$ & $\forall_{x}$ e 3 \\
\hline 8 & & $Q\left(x_{0}\right) \rightarrow R\left(y_{0}\right)$ & $\forall_{y}$ e 7 \\
\hline 9 & & $Q\left(x_{0}\right)$ & $\rightarrow$ e 6,5 \\
\hline 10 & & $R\left(y_{0}\right)$ & $\rightarrow$ e 8,9 \\
\hline 11 & & $R\left(y_{0}\right)$ & $\exists_{x}$ e $1,5-10$ \\
\hline 12 & & $\forall_{y} R(y)$ & $\forall_{y} \mathrm{i} 4-11$ \\
\hline
\end{tabular}

Figure 1: The example of reasoning that is based on the Fitch notation of natural deduction. It justifies the formula $\left(\left(\exists_{x} P(x)\right) \wedge\left(\forall_{x}(P(x) \rightarrow Q(x))\right) \wedge\left(\forall_{x} \forall_{y}(Q(x) \rightarrow R(y))\right)\right) \rightarrow$ $\forall_{y} R(y)$.

we give a unique number for every line (written on the left), and in the justification we write references to all lines that are used. Additionally, we denote by $\rightarrow$ e the Modus Ponens rule, $\frac{\phi \phi \rightarrow \psi}{\psi}$; and by $\forall_{x}$ e the rule for eliminating the universal quantifier $\forall, \frac{\forall_{x} \phi}{\phi[t / x]}$. Rules for eliminating $\exists$ and introducing $\forall$ use boxes to stipulate the scope of an auxiliary variable $x_{0}$ as follows:
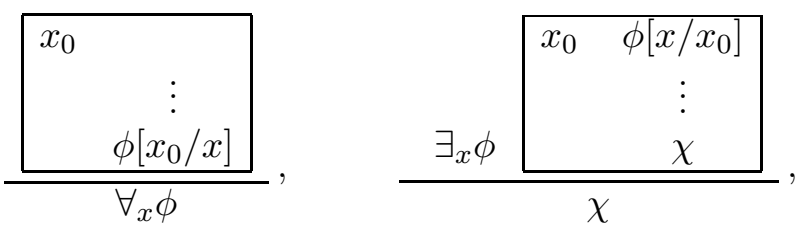

and we denote them by $\forall_{x} \mathrm{i}, \exists_{x} \mathrm{e}$ respectively. The variables $x_{0}$ are called eigenvariables. This means that in case of $\forall_{x} \phi$ it cannot occur in the assumptions of the derivation concluding with $\phi\left[x_{0} / x\right]$ and in case of $\chi$ it cannot occur in $\chi$.

An abstract model of such proofs was considered in [29] for the general case, but for our purposes we consider a simplified model which is represented by a directed acyclic graph (DAG). A DAG $G=\langle V, E\rangle$ with a distinguished set of $\operatorname{arcs} \mathfrak{R}(G) \subseteq E$ is called an abstract proof graph. The vertices of $G$ represent steps of reasoning and $\operatorname{arcs}$ of $G$ represent the flow of information between different steps of reasoning. Additionally, an arc of $\mathfrak{R}(G)$, called a reference arc, describes the dependence between the expression of a step (the head of the 
arc) and a previously justified step (the tail of the arc) used as the justification of that expression. Other arcs of $G$ describe all kinds of additional constraints that force one step to precede another step, e.g. the dependence between steps which introduce variables into the reasoning and steps which use these variables in the expressions.

In general, the reasonings in Jaśkowski-Fitch notation can have nested subreasonings, i.e. ones that are enclosed within boxes created by quantifier rules. The graph model presented here does not take into account this structure. However, the NP-hardness proofs presented in this paper also apply to the appropriate generalization, which includes the nested structure, as they are its special case.

To illustrate the model, let us consider the proof contained in the scope box of $x_{0}$ (Fig. 11). Its model is presented in Fig. 2 where solid arrows represent reference arcs, dashed arrows represent the uses of variable $x_{0}$, and the arrows both solid and dashed represent both reference and the use of a variable. Additionally, both arcs and nodes of the actual abstract proof graph are not labeled (arcs and nodes in Fig. 2, are labeled only to simplify their identification).

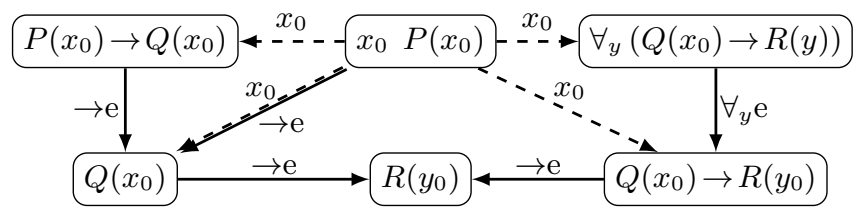

Figure 2: The abstract proof graph illustrating the proof contained in the scope box of $x_{0}$ in Fig. 1 .

We identify here topological sortings of $G$ with one-to-one functions $\tau: V \longrightarrow\{1,2, \ldots,|V|\}$ such that, for every arc $\langle u, v\rangle \in E, \tau(u)<\tau(v)$. Such sortings are sometimes called linearizations. We denote by $T S(G)$ the set of all topological sortings of $G$. We call a vertex of $V$ "then" $\tau$-step if a step of reasoning which corresponds to this vertex uses in justification the immediately preceding step of the derivation induced by $\tau$ (and possibly some other statements). To be more precise, vertex $u$ is a "then" $\tau$-step if and only if for some vertex $v, \tau(v)+1=\tau(u)$ and $\langle v, u\rangle \in \mathfrak{R}(G)$. For example, the step "10 $R\left(y_{0}\right) \rightarrow$ e 8, 9" (Fig. 1) is a "then" step.

To make references to the directly preceding step in a reasoning explicit, we extend the Fitch notation with a new kind of step, called "then" step. Suppose that a step $s$ in line $n+1$ justifies a formula $\phi$ by a rule $\mathfrak{r}$ that refers to the line $n$ and is represented as

$$
n+1 \quad \phi \quad \mathfrak{r} \ldots, n, \ldots,
$$

We present it as a "then" step of the form

$$
n+1 \text { then } \phi \mathfrak{r} \ldots, *, \ldots,
$$

where the number of the previous line $\mathrm{n}$ is replaced by $*$ (see Fig. 3).

It is desirable to have a "push-button" tool that automatically finds a suitable topological sortings $\tau$ of a natural deduction proof so that they have the largest number of "then" $\tau$-steps but we show here that this goal is non-trivial since the problem is NP-complete.

Naturally every sequence of "then" $\tau$-steps determines a linear fragment of reasoning in the proof script that we call a $\tau$-reasoning path. More precisely, let us fix a directed path 


\begin{tabular}{c|cll|}
\cline { 3 - 4 } 5 & $x_{0}$ & $P\left(x_{0}\right)$ & assumption \\
6 & & $P\left(x_{0}\right) \rightarrow Q\left(x_{0}\right)$ & $\forall_{x} \mathrm{e} 2$ \\
9 & then & $Q\left(x_{0}\right)$ & $\rightarrow \mathrm{e} *, 5$ \\
7 & & $\forall_{y}\left(Q\left(x_{0}\right) \rightarrow R(y)\right)$ & $\forall_{x} \mathrm{e} 3$ \\
8 & then & $Q\left(x_{0}\right) \rightarrow R\left(y_{0}\right)$ & $\forall_{y} \mathrm{e} *$ \\
10 & then & $R\left(y_{0}\right)$ & $\rightarrow \mathrm{e} *, 9$ \\
\cline { 2 - 4 }
\end{tabular}

\begin{tabular}{c|cll|}
\cline { 3 - 4 } 5 & $x_{0}$ & $P\left(x_{0}\right)$ & assumption \\
7 & & $\forall_{y}\left(Q\left(x_{0}\right) \rightarrow R(y)\right)$ & $\forall_{x} \mathrm{e} 3$ \\
8 & then & $Q\left(x_{0}\right) \rightarrow R\left(y_{0}\right)$ & $\forall_{y} \mathrm{e} *$ \\
6 & & $P\left(x_{0}\right) \rightarrow Q\left(x_{0}\right)$ & $\forall_{x} \mathrm{e} 2$ \\
9 & then & $Q\left(x_{0}\right)$ & $\rightarrow \mathrm{e} *, 5$ \\
10 & then & $R\left(y_{0}\right)$ & $\rightarrow \mathrm{e} 8, *$ \\
\cline { 2 - 4 }
\end{tabular}

Figure 3: Two possible topological sortings of the proof that is contained in the scope of the $x_{0}$ box in Fig. 1. These sortings have the largest number of "then" steps.

$P=\left\langle u_{0}, u_{1}, u_{2}, \ldots, u_{n}\right\rangle$. Then $P$ is a $\tau$-reasoning path if and only if $u_{k}$ is a "then" $\tau$-step for $k=1,2, \ldots, n$ ( $u_{0}$ does not have to be a "then" $\tau$-step). To say that $P$ is maximal, means simply that $P$ is not a subsequence of any other $\tau$-reasoning path. It is easy to check that the number of "then" $\tau$-steps depends on the number of all maximal $\tau$-reasoning paths. To be more precise, the sum of the number of "then" $\tau$-steps and the number of maximal $\tau$-reasoning paths is constant and equal to the number of reasoning steps. Hence the number of "then" $\tau$-steps is maximal if and only if the number of all maximal $\tau$-reasoning paths is minimal.

This means we can equivalently formulate many definitions in terms of the number of "then" $\tau$-steps and in terms of $\tau$-reasoning paths. In addition, we would like the flow of information between any two different maximal $\tau$-reasoning paths to be minimal. This is equivalent to the property that the number of internal $\tau$-arcs, i.e. the reference arcs whose head and tail belong to the same maximal $\tau$-reasoning path, is maximal. It is true since the sum of all reference arcs between any two different maximal $\tau$-reasoning paths plus the number of internal $\tau$-arcs is constant and equal to the number of reference arcs.

As an illustration of "then" $\tau$-steps and $\tau$-reasoning paths, let us consider a short quasi proof script written in the Mizar style. A theorem in the Mizar style has the form:

$$
\begin{aligned}
& \text { theorem label: statement } \\
& \text { proof } \\
& \text { proof steps } \\
& \text { end; }
\end{aligned}
$$

and a proof step in general has the form:

label: statement by justification ;

Let us focus on a statement of the theory of fields that the inverse of a product of two elements of the field is equal to the product of their inverses. Note that symbols 1.F, 0.F represent the additive identity element and the multiplicative identity element of the field F, respectively. Note additionally that the system Mizar uses only ASCII characters. Operations such as "-1", "." are represented in Mizar as " and *, respectively, and VECTSP_1, GROUP_1, RLVECT_1 are identifiers of Mizar articles [24, 36, 37. The proof script presented in Fig. 4 does not comply well to the Behaghel's First Law. Only one step (line 17) refers to the preceding step, the other steps in the reasoning refer to steps placed far away in the proof script. Analyzing the possible topological sortings of the corresponding proof graph (see Fig. 5) 359598 cases, we get that the greatest number of "then" $\tau$-steps is reached in 16 linearizations and is equal to 12 . Hence the smallest number of maximal $\tau$-reasoning paths is equal to 5 . In 6 cases of these 16, we have also the smallest number of reference arcs between any two different maximal $\tau$-reasoning paths, which is equal to 5 . One of these 6 cases is presented in Fig. 6 . 


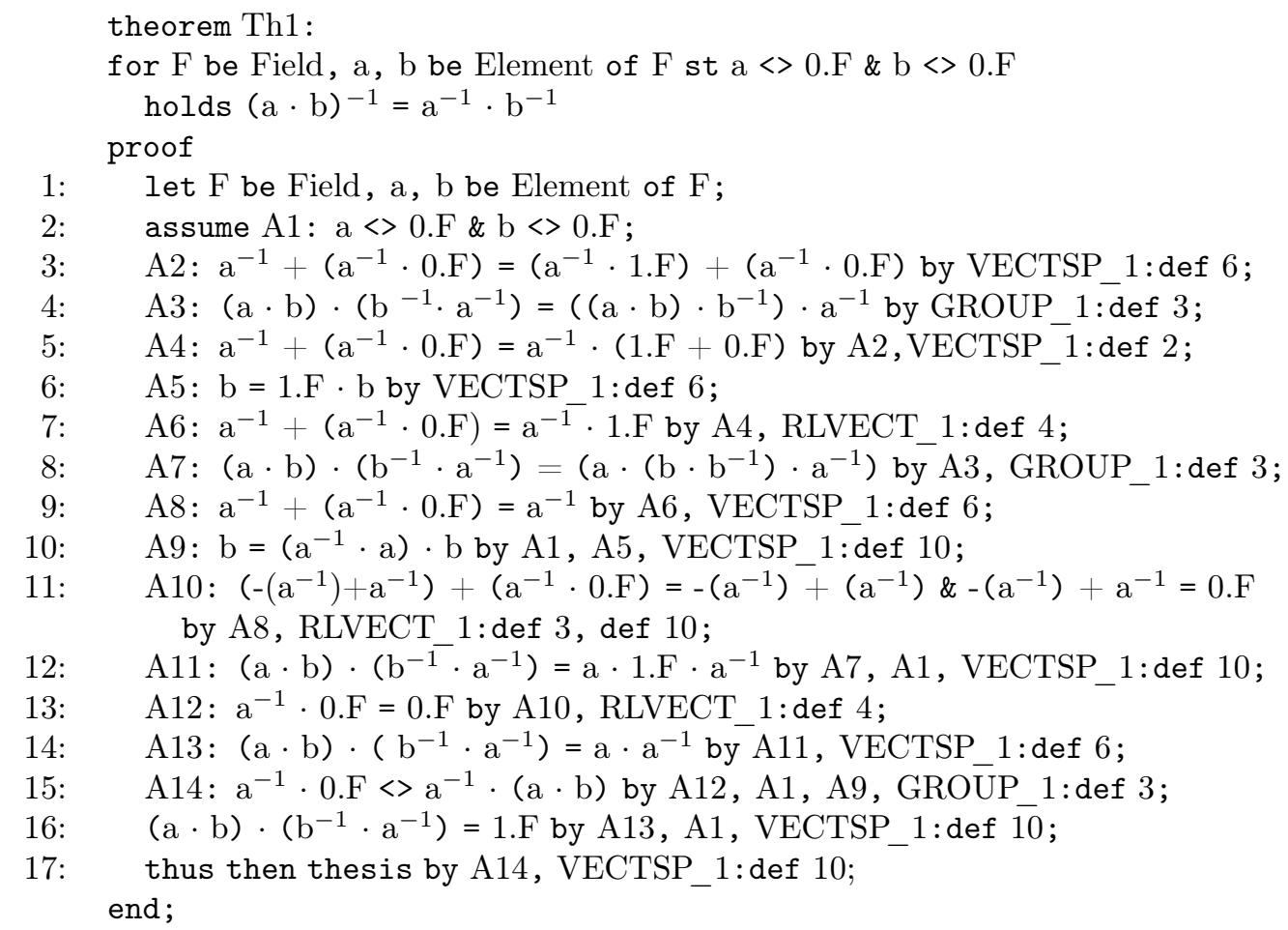

Figure 4: An example of illegible proof script written in the Mizar style.

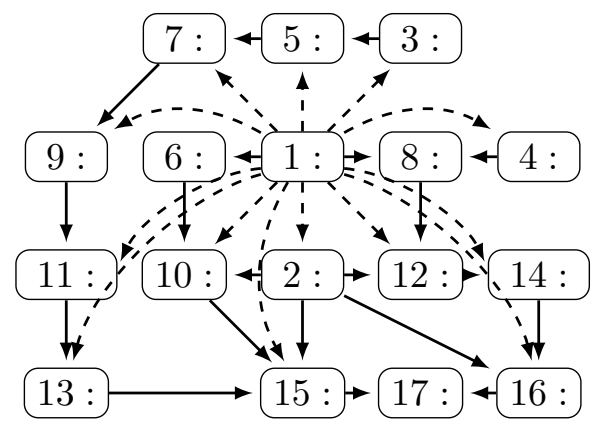

Figure 5: The abstract proof graph illustrating the structure of the reasoning presented in Fig. 4.

This example illustrates the significance of "then" construction in the process of improving proof scripts legibility. This construction in natural way focuses the reader's attention on sequences of "then" $\tau$-steps. Note that every sequence of steps that has a label connected only to the last step of this sequence (as is the case of the sequence of "then" $\tau$-steps starting in step 3 and ending in step 13 in Fig. 6) contains additional information for readers. Naturally, the previous unlabelled steps have only local significance, i.e. each of them is used exclusively to justify the directly following step. This is a sign for readers that they can "encapsulate" the piece of reasoning and abstract away from its content in many situations. 


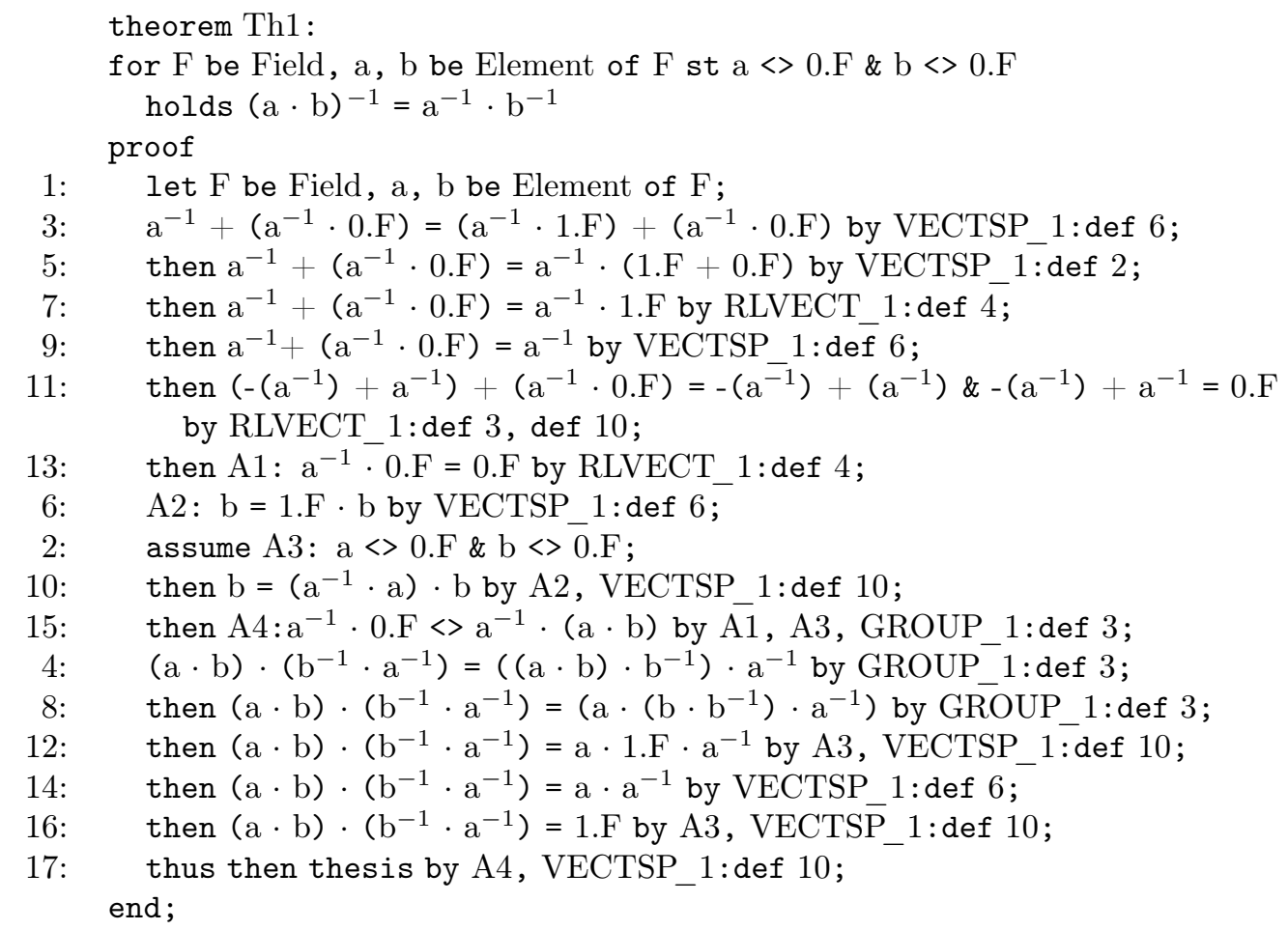

Figure 6: A linearisation of the proof presented in Fig. 4. This linearisation maximizes the number of "then" $\tau$-steps.

The following short analysis of this property shows that it is impossible to apply several proof readability criteria at the same time and that they are sometimes contradictory. A commonly accepted solution in such circumstances is to impose a hierarchy on the optimized properties (this additional property and several others are briefly described in Section 7 ).

Until now, we have concentrated on the number of steps that refer to the preceding step. Maximization of sequences of this kind is not the only strategy to improve readability. The topological sorting presented in Fig. 6 has additional important features that are not consequence of Behaghel's First Law, but are related to readability. In particular, it is one of two linearizations that have the smallest sum of all distances between premises and their uses, equal to 38 (the second topological sorting with this property contains 11 "then" $\tau$ steps). Additionally, 4 labels have been used in this reasoning, but it is not the smallest number of labels in existing linearisation. If we swap lines 6 and 2, then the label A2 is not necessary. Among all sortings of the abstract proof graph in Fig. 5 the smallest possible number of labels is 3 . This is achieved in 8 cases. However, none of the cases minimizes the sum of all distances since the sum there is at best 41 . Notably there are, among the 8 cases, ones that contain exactly 12 "then" $\tau$-steps, which is the maximal possible number for the abstract proof graph. This illustrates that there are proofs for which all plausible readability optimalization criteria cannot be met at once.

Another way to impose a restriction on the distance between premises and their uses is to minimize the maximal distance between an arbitrary premise and its use. This distance for the reasoning presented in Fig. [6 is equal to 7 (the distance is reached between lines 2 and 16 for the label A3). The smallest restriction of maximal distance in this abstract proof 
graph is equal to 5 and is obtained in 128 cases, but optimal values of previously mentioned parameters are not always possible to reach among them. More precisely, the number of "then" $\tau$-steps is at most 12 (the optimal number is 12), the number of reference arcs between any two different maximal $\tau$-reasoning paths is at least 7 (the optimal number is 5 ), the sum of distances between premises and their uses is at least 39 (the optimal number is 38), and the number of labels in a reasoning is at least 6 (the optimal number is 3 ).

\section{Formulation of Behaghel's Law Determinants}

To formulate the criterion we need to set the appropriate vocabulary of notations. Let $G=\langle V, E\rangle$ be a DAG and let $E_{1}$ be a subset of $E$. For a subset $V_{1}$ of $V$ we denote by $G_{\mid V_{1}}$ the subgraph of $G$ induced by $V_{1}$. For a vertex $u$ in $V$, we use the following notation:

$$
\begin{array}{ll}
N_{G}^{-}(u):=\{v \in V:\langle v, u\rangle \in E\} & \text { (incoming arcs), } \\
N_{G}^{+}(u):=\{v \in V:\langle u, v\rangle \in E\} & \text { (outgoing arcs), }
\end{array}
$$

$\left|N_{G}^{-}(u)\right|$ is the in-degree of $u$ and $\left|N_{G}^{+}(u)\right|$ is the out-degree of $u$. An arc is called $E_{1}$-arc if it belongs to $E_{1}$. A path $P=\left\langle u_{1}, u_{2}, \ldots, u_{n}\right\rangle$ of $G$ is called an $E_{1}$-path if $\left\langle u_{i}, u_{i+1}\right\rangle$ is an $E_{1}$-arc for $i=1,2, \ldots, n-1$. The concatenation of two paths $P_{1}, P_{2}$ is denoted by $P_{1} \frown P_{2}$. The length of $P$ is denoted by $l(P)$ and the set $\left\{u_{1}, u_{2}, \ldots, u_{n}\right\}$ of vertices of $P$ is denoted by $\mathcal{V}(P)$. An $E_{1}$-arc $\langle v, u\rangle$ is an $E_{1}$-shortcut if there exists an $E_{1}$-path that leads from $v$ to $u$ and whose length is at last 2 .

Now we define a formal equivalent of a $\tau$-reasoning path, called a $\tau_{E_{1}}$-path. Given $\tau \in T S(G)$, an $E_{1}$-path $P=\left\langle u_{1}, u_{2}, \ldots, u_{n}\right\rangle$ is called a $\tau_{E_{1}}$-path if $\tau\left(u_{i}\right)+1=\tau\left(u_{i+1}\right)$ for $i=1,2, \ldots, n-1$. Additionally, $P$ is said to be a maximal $\tau_{E_{1}}$-path if and only if $\mathcal{V}(P)$ is not included in any other set of vertices of a $\tau_{E_{1}}$-path. Let

$$
\pi_{\tau}^{E_{1}}:=\left\{\mathcal{V}(P) \mid P \text { is a maximal } \tau_{E_{1}} \text {-path }\right\}
$$

and call it a partition of $G$ determined by $\tau$ with respect to $E_{1}$. For subsets $V_{1}, V_{2}$ of $V$ we use the following notation:

$$
V_{1} \underset{E_{1}}{\curvearrowright} V_{2}:=\left\{\left\langle v_{1}, v_{2}\right\rangle \in E_{1} \mid v_{1} \in V_{1} \wedge v_{2} \in V_{2}\right\} .
$$

The methods of improving legibility of proofs described above can be formulated as the following two decision problems:

\section{1st Method of Improving Legibility (1st MIL):}

Instance: A DAG $G=\langle V, E\rangle$, a subset $E_{1}$ of $E$, and a positive integer $K \leq|V|$.

Question: Does there exist a topological sorting $\tau$ of $G$ for which $\pi_{\tau}^{E_{1}}$ has size at most $K$ ?

\section{2nd Method of Improving Legibility (2nd MIL):}

Instance: A DAG $G=\langle V, E\rangle$, a subset $E_{1}$ of $E$, and a positive integer $L \leq\left|E_{1}\right|$.

QuESTIOn: Does there exist a topological sorting $\tau$ of $G$ for which

$$
\sum_{\substack{P_{1}, P_{2} \in \pi_{\tau}^{E_{1}} \\ P_{1} \neq P_{2}}}\left|P_{1} \underset{E_{1}}{\curvearrowright} P_{2}\right| \leq L ?
$$


In our setting, the subset $E_{1}$ corresponds to the set of reference arcs. The 1st MIL corresponds to searching for a topological sorting $\tau$ that has the smallest possible number of maximal sequences of "then" $\tau$-steps. Recall that we can formulate this problem in the equivalent way as maximization of the number of "then" $\tau$-steps.

The 2nd MIL is an extension of the idea of the first method. We demand that every "then" $\tau$-step not only refers to the preceding step in a maximal sequence of "then" $\tau$-steps, but also that other references of this step point mostly to steps of this sequence. To fulfil this condition we search for a topological sorting $\tau$ that has at most a given number of references between any two different maximal sequences of "then" $\tau$-steps.

Now we show that these problems have the same answer for a special type of DAGs and parameters $K, L$ such that $K=L+|V|-\left|E_{1}\right|$.

Lemma 3.1. Let $G=\langle V, E\rangle$ be a $D A G$ and $E_{1}$ be a subset of $E$ for which $G$ is without $E_{1}$-shortcuts. For any $\tau \in T S(G)$, the following holds:

$$
|V|-\left|\pi_{\tau}^{E_{1}}\right|=\left|E_{1}\right|-\sum_{\substack{P_{1}, P_{2} \in \pi_{\tau}^{E_{1}} \\ P_{1} \neq P_{2}}}\left|P_{1} \underset{E_{1}}{\curvearrowright} P_{2}\right| .
$$

Proof. Since $G$ is without $E_{1}$-shortcuts, we have $|P|-1=\left|P \underset{E_{1}}{\curvearrowright} P\right|$ for $P \in \pi_{\tau}^{E_{1}}$. Consequently, $\left|E_{1}\right|=\sum_{\substack{P_{1}, P_{2} \in \pi_{\tau}^{E_{1}} \\ P_{1} \neq P_{2}}}\left|P_{1} \underset{E_{1}}{\curvearrowright} P_{2}\right|+\sum_{P \in \pi_{\tau}^{E_{1}}}(|P|-1)$, but $\sum_{P \in \pi_{\tau}^{E_{1}}}(|P|-1)=|V|-\left|\pi_{\tau}^{E_{1}}\right|$.

This lemma makes it possible to give a single construction that shows hardness of both problems. We exploit it later in the next section.

\section{Acyclic Hamiltonian Partition}

The notion of topological sorting is difficult to manipulate and makes various formulations obscure. Therefore it is useful to express these problems in terms of acyclic partitions. Following Borowiecki and Mihók [6] we use the following notation. Let $\mathcal{P}$ be a graph property. A $\mathcal{P}^{k}$-partition of a graph $G=\langle V, E\rangle$ is a partition $\pi=\left\{V_{1}, V_{2}, \ldots, V_{k}\right\}$ of $V$ such that $G_{\mid V_{i}}$ has property $\mathcal{P}$ for $i=1,2, \ldots, k$. A partition $\phi$ of $G$ is called a $\mathcal{P}^{*}$-partition if there exists a positive integer $k$ such that $\phi$ is a $\mathcal{P}^{k}$-partition. Let us denote by $\mathcal{G}(G, \pi)$ the directed graph $\left\langle\pi,\left\{\left\langle V_{i}, V_{j}\right\rangle: 1 \leq i, j \leq k \wedge i \neq j \wedge V_{i} \underset{E}{\curvearrowright} V_{j} \neq \emptyset\right\}\right\rangle$. We say that $\mathcal{P}^{k}$-partition $\pi$ is acyclic if and only if $\mathcal{G}(G, \pi)$ is acyclic. For simplicity, an acyclic $\mathcal{P}^{k}$-partition is called a $\mathcal{P}^{(k)}$-partition and an acyclic $\mathcal{P}^{*}$-partition is called a $\mathcal{P}^{(*)}$-partition. Let $G=\langle V, E\rangle$ be a DAG and let $V_{1}$ be a subset of $V$. We define the property $\mathcal{H}$ of $G$ as: the subgraph $G_{\mid V_{1}}$ has a Hamiltonian path.

Our main object of investigation is now the following problem:

Acyclic Hamiltonian Partition (AHP):

Instance: A DAG $G=\langle V, E\rangle$ and a positive integer $K \leq|V|$.

QuESTION: Is there an $\mathcal{H}^{(*)}$-partition of $G$ of size at most $K$ ?

It is evident that $\pi_{\tau}^{E}$ is an $\mathcal{H}^{\left(\left|\pi_{\tau}^{E}\right|\right)}$-partition of $G$ for any $\tau \in T S(G)$. We show that an $\mathcal{H}^{(*)}$-partition $\pi$ of $G$ which solves AHP determines the topological sorting $\tau$ of $G$ such 
that $\left|\pi_{\tau}^{E}\right| \leq|\pi|$. In consequence we can consider the 1st MIL problem to be equivalent to AHP in the case $E=E_{1}$. Hence if we show that AHP is an NP-complete problem for DAG without $E$-shortcuts, then 1 st MIL is also NP-complete for DAG without $E_{1}$-shortcuts, where $E_{1}=E$. Additionally for so restricted instances, 1st MIL and 2nd MIL are equivalent by Lemma 3.1. Based on this observation, to show that these methods are NP-complete, we focus in Section 5 only on the complexity of AHP.

To shorten our notation, $\mathfrak{h}^{\pi}(p)$ denotes the Hamiltonian path of $G_{\mid p}$, where $p \in \pi$ and $\pi$ is an $\mathcal{H}^{*}$-partition. Additionally, we denote by $\mathfrak{h}^{\pi}(v)$ the path $\mathfrak{h}^{\pi}(p)$, where $v \in p$.

Lemma 4.1. Let $\pi$ be an $\mathcal{H}^{(*)}$-partition and suppose that $\pi$ solves AHP. Then there exists $\varrho \in T S(G)$ such that $\left|\pi_{\varrho}^{E}\right| \leq|\pi|$.

Proof. As $\pi$ is acyclic we can take $\tau \in T S(\mathcal{G}(G, \pi))$. Let $\mathfrak{h}^{\pi}(P)=\left(u_{1}^{P}, u_{2}^{P}, \ldots, u_{|P|}^{P}\right)$, for $P \in \pi$. Let $\varrho: \mathcal{V}(G) \rightarrow\{1,2, \ldots,|\mathcal{V}(G)|\}$ be a function given by the formula $\varrho\left(u_{i}^{Q}\right)=$ $i+\sum_{R \in \pi: \tau(R)<\tau(Q)}|R|$, where $1 \leq i \leq|Q|, Q \in \pi$. Observe that $\varrho$ is a topological sorting of $G=\langle V, E\rangle$. Obviously, every Hamiltonian path $\mathfrak{h}^{\pi}(P)$ is also a $\varrho_{E}$-path, for $P \in \pi$. Therefore, the number of all maximal $\varrho_{E}$-paths is not greater than the cardinality of $\pi$.

\section{The NP-COMPleteness of the AHP PROBLEM}

It is clear that AHP is in NP. We can guess a partition and verify that it is indeed an $\mathcal{H}^{(*)}$-partition. We give here a decision problem for directed graphs and its transformation to AHP that proves that AHP is NP-hard. Given $G=\langle V, E\rangle$, a feedback arc set is a subset $E^{\prime}$ of $E$ that contains at least one arc from each directed cycle of $G$. The following problems are NP-complete (see GT1, GT8 in [10]).

Feedback Arc Set (FAS):

InstANCE: A directed graph $G=\langle V, E\rangle$ and a positive integer $K \leq|V|$.

QUESTION: Is there a feedback arc set of $G$ of size at most $K$ ?

\section{Vertex Cover (VC):}

InSTANCE: An undirected graph $G=\langle V, E\rangle$ and a positive integer $K \leq|V|$.

Question: Is there a vertex cover of size at most $K$, i.e. a subset $V^{\prime} \subseteq V$ with $\left|V^{\prime}\right| \leq K$ such that for each edge $\{u, v\} \in E$ at least one of $u$ or $v$ belongs to $V^{\prime}$ ?

Transformation of VC to FAS has been considered by R. M. Karp [21]. Using this transformation we can prove that FAS is NP-complete even for digraphs in which all vertices have in-degree or out-degree equal to 1.

Lemma 5.1. FAS is NP-complete even for directed graphs without self-loops, in which additionally all vertices have in-degree or out-degree equal to 1.

Proof. Given an undirected graph $G=\langle V, E\rangle$, we follow R. M. Karp and construct the directed graph $G^{\prime}=\left\langle V^{\prime}, E^{\prime}\right\rangle$ as

$$
\begin{aligned}
& V^{\prime}=V \times\{0,1\}, \\
& E^{\prime}=\{\langle\langle v, 0\rangle,\langle v, 1\rangle\rangle: v \in V\} \cup\{\langle\langle u, 1\rangle,\langle v, 0\rangle\rangle:\{u, v\} \in E\} .
\end{aligned}
$$


There are no self-loops as for $\langle\langle u, i\rangle,\langle v, j\rangle\rangle \in E^{\prime}$ we have $i \neq j$. The out-degree of vertices $\langle u, 0\rangle$ is 1 and in-degree of vertices $\langle v, 1\rangle$ is 1 . The directed graph $G^{\prime}$ has the property formulated in the proposition.

We transform the subcase of FAS that is specified in Lemma 5.1 to AHP. Let $G=\langle V, E\rangle$ be a directed graph without self-loops, in which additionally all vertices have in-degree or out-degree equal to 1 . The case $V=\emptyset$ is obvious, therefore we can assume that $|V|>0$. Note that since there exists at last one vertex with in-degree or out-degree equal to 1 , we have $|E|>0$. Let us fix a one-to-one function $e: E \longrightarrow\{1,2, \ldots,|E|\}$. We construct a DAG $\mathcal{P}(G, e)=\left\langle V_{G}, E_{G}\right\rangle$ without $E_{G}$-shortcuts (Def. 5.5) and we prove that there exists $\mathcal{F}$ that is a feedback arc set of $E$ which has size at most $K$ if and only if there exists an $\mathcal{H}^{(*)}$-partition $\pi_{\mathcal{F}}$ of $\mathcal{P}(G, e)$ with size at most $|V| \cdot(|E|+1)+K$. The set $V_{G}$ of vertices is divided in two separate classes, grouped according to their intended function: "gadgets", each of which corresponds to a single vertex of $V$, and "glues", which correspond to a single arc from $E$. We divide this construction in sequence of steps and statements, included into three subsections. In Subsection 5.1 we introduce the notion of gadgets and prove their essential properties. Next in Subsection 5.2 we complete the definition of $\mathcal{P}(G, e)$ by presentation of "glues" and construct $\pi_{\mathcal{F}}$ using $\mathcal{F}$. At the end of Subsection 5.3 we construct the feedback arc set $\mathcal{F}$ using $\pi_{\mathcal{F}}$.

Let us fix the notation $\mathcal{G}=\langle\mathbb{V}, \mathbb{E}\rangle$ for a directed graph without self-loops, in which all vertices have in-degree or out-degree equal to 1.

5.1. The Gadgets. Let us take a vertex $r \in \mathbb{V}$. We call a graph $\mathcal{N}_{r}=\left\langle\mathcal{V}_{r}, \mathcal{E}_{r}\right\rangle$ (see Fig. 7) the gadget corresponding to $r$, and define it as:

$$
\begin{aligned}
& \mathcal{V}_{r}=\left\{r_{i, j}: 0 \leq i, j \leq|\mathbb{E}|\right\}, \\
& \mathcal{E}_{r}=\swarrow_{r} \cup \searrow_{r},
\end{aligned}
$$

where

$$
\begin{aligned}
\swarrow_{r}:= & \left\{\left\langle r_{i, j}, r_{i, j+1}\right\rangle: 0 \leq i, j<|\mathbb{E}|\right\} \cup\left\{\left\langle r_{|\mathbb{E}|, i}, r_{|\mathbb{E}|, i+1}\right\rangle: 0 \leq i<|\mathbb{E}|\right\}, \\
\searrow_{r}:= & \left\{\left\langle r_{i, j}, r_{i+1, j}\right\rangle: 0 \leq i, j<|\mathbb{E}|\right\} \cup\left\{\left\langle r_{i,|\mathbb{E}|}, r_{i+1,|\mathbb{E}|}\right\rangle: 0 \leq i<|\mathbb{E}|\right\} .
\end{aligned}
$$

Let us introduce notations for $\mathcal{E}_{r}$-paths:

$$
\swarrow_{r}^{i}:=\left(r_{i, 0}, r_{i, 1}, \ldots, r_{i,|\mathbb{E}|}\right), \quad \searrow_{r}^{i}:=\left(r_{0, i}, r_{1, i}, \ldots, r_{|\mathbb{E}|, i}\right),
$$

where $0 \leq i \leq|\mathbb{E}|$, and for $\mathcal{H}^{(|\mathbb{E}|+1)}$-partitions of $\mathcal{N}_{r}$ :

$$
\mathcal{L}_{r}:=\left\{\mathcal{V}\left(\swarrow_{r}^{i}\right): 0 \leq i \leq|\mathbb{E}|\right\}, \quad \mathcal{R}_{r}:=\left\{\mathcal{V}\left(\searrow_{r}^{i}\right): 0 \leq i \leq|\mathbb{E}|\right\} .
$$

For simplicity of notation an $\mathcal{E}_{r}$-path $P$ is denoted by $\swarrow_{r}^{*}$ if there exists a positive integer $i$ such that $P=\swarrow_{r}^{i}$, and analogously we denote by $\searrow_{r}^{*}$ an $\mathcal{E}_{r}$-path $P$ if there exists a positive integer $j$ such that $P=\searrow_{r}^{j}$.

Obviously $\mathcal{N}_{r}$ is a DAG without $\mathcal{E}_{r}$-shortcuts, since an arbitrary $\mathcal{E}_{r}$-arc $\left\langle r_{i_{1}, j_{1}}, r_{i_{2}, j_{2}}\right\rangle$ fulfils the condition $i_{1}+j_{1}+1=i_{2}+j_{2}$. Moreover, a set of $\mathcal{E}_{r}$-paths that can determine an $\mathcal{H}^{(*)}$-partition of $\mathcal{N}_{r}$ is included only in paths that are subsequences of $\swarrow_{r}^{*}$ or $\searrow_{r}^{*}$. It is expressed more precisely in the following lemma.

Lemma 5.2. Let $\pi$ be an $\mathcal{H}^{(*)}$-partition of $\mathcal{N}_{r}$ and $P \in \pi$. Then the path $\mathfrak{h}^{\pi}(P)$ has arcs in $\swarrow_{r}$ only or in $\searrow_{r}$ only. 


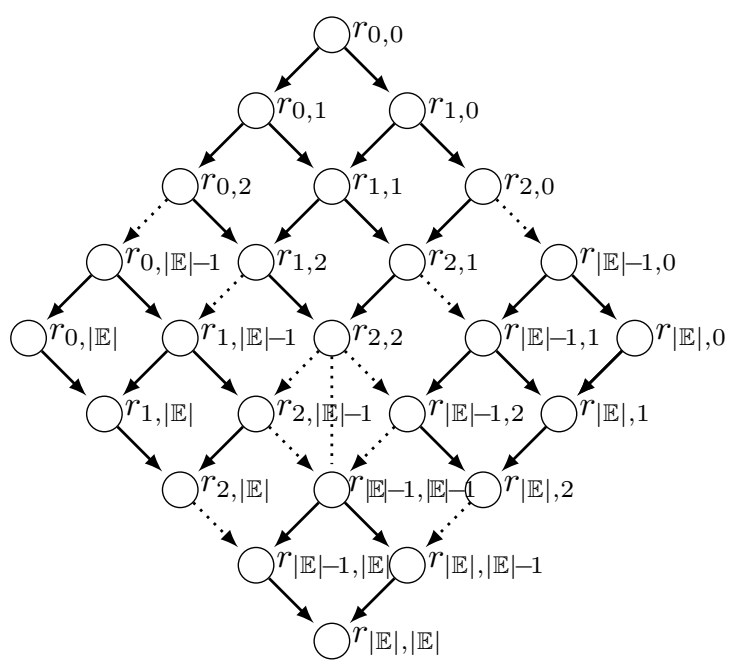

Figure 7: The gadget $\mathcal{N}_{r}$.

Proof. The proof is by contradiction. Suppose that $\mathfrak{h}^{\pi}(P)$ uses at least one $\swarrow r^{\text {-arc }}$ and at least one $\searrow_{r}$-arc. Note that for each consecutive elements of this path $r_{i_{1}, j_{1}}, r_{i_{2}, j_{2}}$

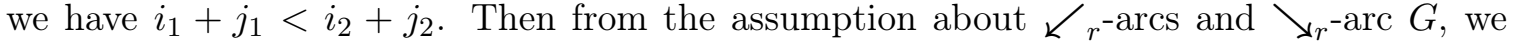
obtain that $\mathfrak{h}^{\pi}(P)$ has $\left\langle r_{i, j}, r_{i, j+1}\right\rangle,\left\langle r_{i, j+1}, r_{i+1, j+1}\right\rangle$ arcs or $\left\langle r_{i, j}, r_{i+1, j}\right\rangle,\left\langle r_{i+1, j}, r_{i+1, j+1}\right\rangle$, for some $0 \leq i, j<|\mathbb{E}|$. We prove only the first case, the second case is analogous. Since $i+1+j<$ $i+1+j+1<k+l$ for each $r_{k, l} \in P$ after $r_{i+1, j+1}$ and $i+1+j>i+j>k+l$ for each $r_{k, l} \in P$ before $r_{i, j}$, we obtain that $\mathfrak{h}^{\pi}(P) \neq \mathfrak{h}^{\pi}\left(r_{i+1, j}\right)$. Then $\left\langle P, \mathcal{V}\left(\mathfrak{h}^{\pi}\left(r_{i+1, j}\right)\right)\right\rangle,\left\langle\mathcal{V}\left(\mathfrak{h}^{\pi}\left(r_{i+1, j}\right)\right), P\right\rangle$ are arcs in $\mathcal{G}\left(\mathcal{N}_{r}^{n}, \pi\right)$, which generates a directed cycle in the acyclic graph $\mathcal{G}\left(\mathcal{N}_{r}^{n}, \pi\right)$. This is a contradiction with the assumption that $\pi$ is an $\mathcal{H}^{(*)}$-partition.

We can now prove that either parts of all $\swarrow_{r}^{i}$ or all $\searrow_{r}^{i}$ can be found in an $\mathcal{H}^{(*)}$-partition.

Lemma 5.3. Let $\pi$ be an $\mathcal{H}^{(*)}$-partition of $\mathcal{N}_{r}$. Then for each $i \in\{0,1, \ldots,|\mathbb{E}|\}$ there exists $L_{i} \in \pi$ such that $\mathfrak{h}^{\pi}\left(L_{i}\right)$ is a subsequence of $\swarrow_{r}^{i}$ or for each $i \in\{0,1, \ldots,|\mathbb{E}|\}$ there exists $R_{i} \in \pi$ such that $\mathfrak{h}^{\pi}\left(R_{i}\right)$ is a subsequence of $\searrow_{r}^{i}$.

Proof. Suppose that there exists $i, 0 \leq i \leq|\mathbb{E}|$, such that for every $L \in \pi, \mathfrak{h}^{\pi}(L)$ is not a subsequence of $\swarrow_{r}^{i}$. Then from Lemma 5.2 we conclude that $\mathfrak{h}^{\pi}\left(r_{i, 0}\right), \mathfrak{h}^{\pi}\left(r_{i, 1}\right), \ldots, \mathfrak{h}^{\pi}\left(r_{i,|\mathbb{E}|}\right)$ are pairwise different paths and $\mathfrak{h}^{\pi}\left(r_{i, j}\right)$ is a subsequence of $\searrow_{r}^{j}$, for $j=0,1, \ldots,|\mathbb{E}|$, which completes the proof.

An easy computation shows that from Lemma 5.2 and 5.3 we can infer the following lemma.

Lemma 5.4. An arbitrary $\mathcal{H}^{(*)}$-partition of $\mathcal{N}_{r}$ has size at least $|\mathbb{E}|+1$. Moreover, there exist exactly two $\mathcal{H}^{(|\mathbb{E}|+1)}$-partitions of $\mathcal{N}_{r}$, they are $\mathcal{L}_{r}, \mathcal{R}_{r}$.

5.2. The DAG $\mathcal{P}(\mathcal{G}, e)$. In the construction of the digraph $\mathcal{P}(\mathcal{G}, e)$ we use a one-to-one function $e$, but its choice does not affect the properties of the considered digraph. 
Definition 5.5. Let $e: \mathbb{E} \longrightarrow\{1,2, \ldots,|\mathbb{E}|\}$ be a one-to-one function. We define a graph $\mathcal{P}(\mathcal{G}, e):=\left\langle\mathbb{V}_{\mathcal{G}}, \mathbb{E}_{\mathcal{G}}\right\rangle$ (see Fig. [) as:

$$
\begin{array}{r}
\mathbb{V}_{\mathcal{G}}=\bigcup_{v \in \mathbb{V}} \mathcal{V}_{v} \cup \mathbb{E}, \\
\mathbb{E}_{\mathcal{G}}=\bigcup_{v \in \mathbb{V}} \mathcal{E}_{v} \cup\left\{\left\langle\langle v, u\rangle, v_{e(\langle v, u\rangle), 0}\right\rangle:\langle v, u\rangle \in \mathbb{E}\right\} \cup \\
\quad\left\{\left\langle\langle v, u\rangle, u_{0, e(\langle v, u\rangle)}\right\rangle:\langle v, u\rangle \in \mathbb{E}\right\} .
\end{array}
$$

Clearly, $\mathcal{P}(\mathcal{G}, e)_{\mid \mathcal{V}_{v}}=\mathcal{N}_{v}$ for every $v \in \mathbb{V}$.

In the following considerations, we construct an $\mathcal{H}^{(*)}$-partition $\pi(\mathcal{F})$ of $\mathcal{P}(\mathcal{G}, e)$ for a given feedback arc set $\mathcal{F}$ of $\mathcal{G}$, which fulfils $|\pi(\mathcal{F})|=|\mathbb{V}| \cdot(|\mathbb{E}|+1)+|\mathcal{F}|$. Note that throughout this construction the size of in-degree and out-degree in $\mathcal{G}$ is not used.
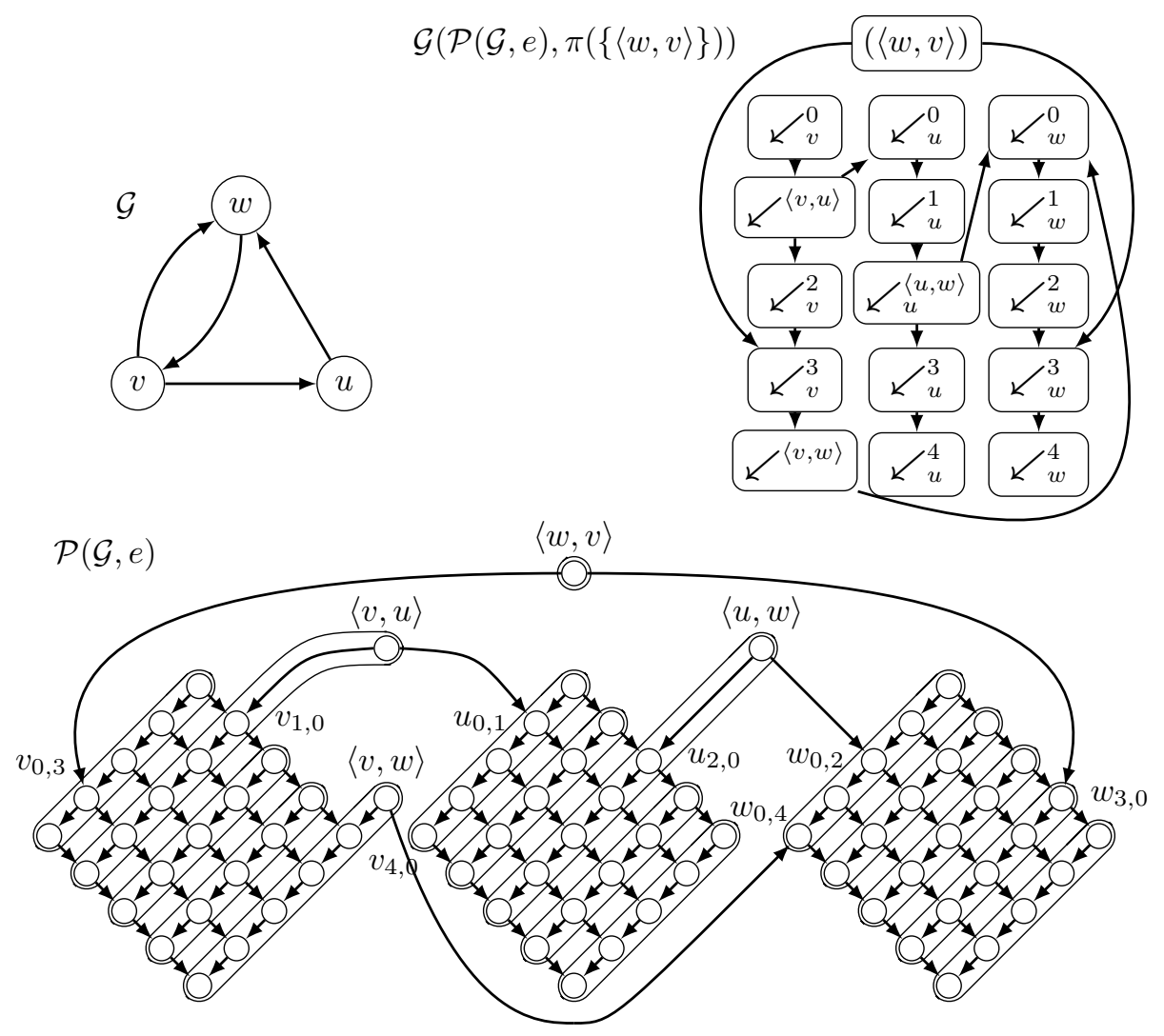

Figure 8: The $\mathcal{H}^{(16)}$-partition $\pi(\mathcal{F})$ of $\mathcal{P}(\mathcal{G}, e)$, an example that illustrates the construction from the proof of Lemma 5.6, where $e(\langle v, u\rangle)=1, e(\langle u, w\rangle)=2, e(\langle w, v\rangle)=3$, $e(\langle v, w\rangle)=4, \mathcal{F}=\{\langle w, v\rangle\}$.

Lemma 5.6. Let $\mathcal{F}$ be a feedback arc set of $\mathcal{G}$. Then there exists an $\mathcal{H}^{(|\mathbb{V}| \cdot(|\mathbb{E}|+1)+|\mathcal{F}|)}$-partition of $\mathcal{P}(\mathcal{G}, e)$. 
Proof. We define a partition $\pi(\mathcal{F})$ of $\mathbb{V}_{\mathcal{G}}$ determined by $\mathcal{F}$ as:

$$
\begin{aligned}
& \pi(\mathcal{F})=\left\{\left\{e^{-1}(i), v_{i, 0}, v_{i, 1}, \ldots, v_{i,|\mathbb{E}|}\right\}:\right. \\
&\left.v \in \mathbb{V} \wedge 0 \leq i \leq|\mathbb{E}| \wedge \underset{u \in \mathbb{V}}{\exists}\left(e^{-1}(i)=\langle v, u\rangle \wedge\langle v, u\rangle \in \mathbb{E} \backslash \mathcal{F}\right)\right\} \cup \\
&\left\{\left\{v_{i, 0}, v_{i, 1}, \ldots, v_{i,|\mathbb{E}|}\right\}:\right. \\
&\left.v \in \mathbb{V} \wedge 0 \leq i \leq|\mathbb{E}| \wedge \neg \underset{u \in \mathbb{V}}{\exists}\left(e^{-1}(i)=\langle v, u\rangle \wedge\langle v, u\rangle \in \mathbb{E} \backslash \mathcal{F}\right)\right\} \cup \\
& \\
&\{\{\langle v, u\rangle\}:\langle v, u\rangle \in \mathcal{F}\} .
\end{aligned}
$$

Elements of $\pi(\mathcal{F})$ can be divided into three types of $\mathbb{V}_{\mathcal{G}}$-paths:

(1) $\left\langle\langle v, u\rangle \swarrow_{\swarrow_{v}^{e}}^{e(\langle v, u\rangle)}\right.$, if $\langle v, u\rangle \in \mathbb{E} \backslash \mathcal{F}$ (denoted by $\swarrow^{\langle v, u\rangle}$ ),

(2) $\swarrow_{v}^{i}$, if $\neg \underset{u \in \mathbb{V}}{\exists}\left(e^{-1}(i)=\langle v, u\rangle \wedge\langle v, u\rangle \in \mathbb{E} \backslash \mathcal{F}\right)$,

(3) $\langle\langle v, u\rangle\rangle$, if $\langle v, u\rangle \in \mathcal{F}$.

It is easy to check that $\pi(\mathcal{F})$ is an $\mathcal{H}^{|\mathbb{V}| \cdot(|\mathbb{E}|+1)+|\mathcal{F}|}$-partition of $\mathcal{P}(\mathcal{G}, e)$. To complete the proof it is enough to show only that $\mathcal{G}(\mathcal{P}(\mathcal{G}, e), \pi(\mathcal{F}))$ is acyclic.

Suppose, contrary to our claim, that there exists a cycle $\mathfrak{a}=\left\langle a_{1}, a_{2}, \ldots, a_{k}\right\rangle$ in $\mathcal{G}(\mathcal{P}(\mathcal{G}, e), \pi(\mathcal{F}))$, where $k>2$ and $a_{k}=a_{1}$. Since all paths of the 3rd type correspond to a source in $\mathcal{G}(\mathcal{P}(\mathcal{G}, e), \pi(\mathcal{F}))$, see (5.6) , we conclude that for each $i \in\{1,2, \ldots, k-1\}$ there is a vertex $v$ such that $a_{i} \cap \mathcal{V}_{v} \neq \emptyset$. Moreover this vertex is unique for each $i$. Let $v_{i}$ be such vertex corresponding to $a_{i}$. Obviously, vertices $v_{1}, v_{2}, \ldots, v_{k-1}$ do not have to be pairwise different. However let us consider the maximal subsequence $\left\langle v_{1}^{\prime}, v_{2}^{\prime}, \ldots, v_{k^{\prime}-1}^{\prime}, v_{k^{\prime}}^{\prime}\right\rangle$ of $\left\langle v_{1}, v_{2}, \ldots, v_{k-1}, v_{1}\right\rangle$ such that $v_{i}^{\prime} \neq v_{i+1}^{\prime}$ for $i=1,2, \ldots, k^{\prime}-1$ where $v_{k}^{\prime}=v_{1}^{\prime}$. We prove that this subsequence is a cycle of the graph $\langle\mathbb{V}, \mathbb{E} \backslash \mathcal{F}\rangle$ which contradicts the fact $\mathcal{F}$ is a feedback arc set of $\mathcal{G}$.

For this purpose let us consider vertices $a_{i}, a_{i+1}$ of $\mathcal{G}(\mathcal{P}(\mathcal{G}, e), \pi(\mathcal{F}))$ which satisfy $v_{i} \neq$ $v_{i+1}$. Obviously, both vertices $a_{i}, a_{i+1}$ have to correspond to $\mathbb{V}_{\mathcal{G}}$-paths of [1st or 2 nd type. From this, it is easy to check that since $\left\langle a_{i}, a_{i+1}\right\rangle$ is an $\operatorname{arc}$ of $\mathcal{G}(\mathcal{P}(\mathcal{G}, e), \pi(\mathcal{F})$ ), we have $a_{i}=\mathcal{V}\left(\swarrow^{\left\langle v_{i}, v_{i+1}\right\rangle}\right), a_{i+1}=\mathcal{V}\left(\swarrow_{v_{i+1}}^{0}\right)$, and the form of $a_{i}$ implies that $\left\langle v_{i}, v_{i+1}\right\rangle \in \mathbb{E} \backslash \mathcal{F}$.

5.3. A feedback arc set of $\mathcal{G}$ determined by an $\mathcal{H}^{(*)}$-partition of $\mathcal{P}(\mathcal{G}, e)$. Let us consider an $\mathcal{H}^{(*)}$-partition $\pi$ of $\mathcal{P}(\mathcal{G}, e)$. First we show in Lemma 5.8 that there exists an $\mathcal{H}^{(*)}$-partition $\pi^{\prime}$ of $\mathcal{P}(\mathcal{G}, e)$ which satisfies $\left|\pi^{\prime}\right| \leq|\pi|$ and additionally has the property that for each $v \in \mathbb{V}: \pi^{\prime}$ restricted to the gadget $\mathcal{N}_{v}$ is a $\mathcal{L}_{v}$ or $\mathcal{R}_{v}$-partition. Since every gadget $\mathcal{N}_{v}$ in $\pi^{\prime}$ is a $\mathcal{L}_{v}$ or $\mathcal{R}_{v}$-partition, we construct in Lemma 5.9 a feedback arc set of $\mathcal{G}$ which has size at most $|\pi|-|\mathbb{V}| \cdot(|\mathbb{E}|+1)$.

Let $\pi$ be an $\mathcal{H}^{(*)}$-partition of $\mathcal{P}(\mathcal{G}, e)$. For a vertex $v \in \mathbb{V}$ we use the following notations: $\pi_{\mid v}:=\left\{\mathcal{V}\left(\mathfrak{h}^{\pi}(w)\right): w \in \mathcal{V}_{v}\right\}, \pi_{\mid \mathcal{N}_{v}}:=\left\{\mathcal{V}\left(\mathfrak{h}^{\pi}(w)\right) \cap \mathcal{V}_{v}: w \in \mathcal{V}_{v}\right\}, \mathcal{P}(\mathcal{G}, e)_{\mid \pi, v}:=\mathcal{P}(\mathcal{G}, e)_{\mid \bigcup \pi_{\mid v}}$. Obviously $\pi_{\mid \mathcal{N}_{v}}$ is an $\mathcal{H}^{(*)}$-partition of $\mathcal{N}_{v}$. We also denote by $\mathbb{V}_{\mathcal{G} \mid \pi, v}$ the set of vertices of $\mathcal{P}(\mathcal{G}, e)_{\mid \pi, v}$, and by $\mathbb{E}_{\mathcal{G} \mid \pi, v}$ the set of $\operatorname{arcs}$ of $\mathcal{P}(\mathcal{G}, e)_{\mid \pi, v}$.

Definition 5.7. Let $\pi$ be an $\mathcal{H}^{(*)}$-partition of $\mathcal{P}(\mathcal{G}, e)$ and $v \in \mathbb{V}$. We call a gadget $\mathcal{N}_{v}$ well-oriented in $\pi$ (see Fig. 9) if and only if $\pi_{\mid \mathcal{N}_{v}}=\mathcal{L}_{v}$ or $\pi_{\mid \mathcal{N}_{v}}=\mathcal{R}_{v}$. In the case $\pi_{\mid \mathcal{N}_{v}}=\mathcal{L}_{v}$ we say that the gadget $\mathcal{N}_{v}$ is $\swarrow$-oriented in $\pi$, and $\searrow$-oriented in $\pi$, in the second case.

Lemma 5.8. Let $\pi$ be an $\mathcal{H}^{(*)}$-partition of $\mathcal{P}(\mathcal{G}, e)$ for which the gadget $\mathcal{N}_{u}$ is not welloriented where $u \in \mathbb{V}$. Then there exists an $\mathcal{H}^{(*)}$-partition $\pi^{\prime}$ of $\mathcal{P}(\mathcal{G}, e)$ which satisfies 

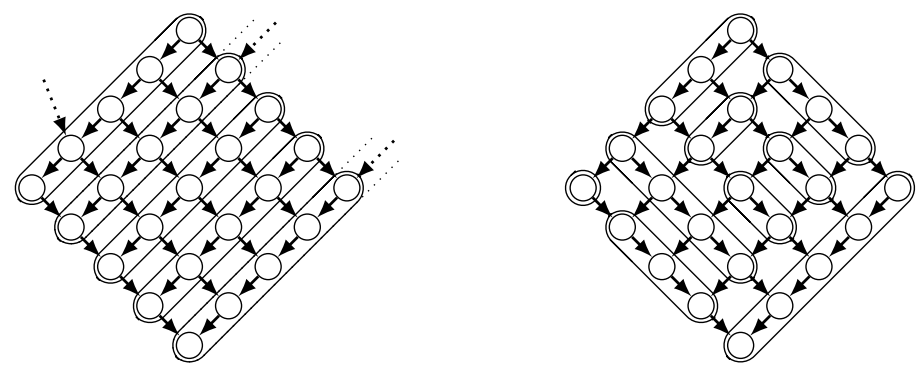

Figure 9: Part of the graph $\mathcal{P}(\mathcal{G}, e)$ presented in Fig. 8 that illustrates the $\mathcal{H}^{(*)}$-partition $\pi(\mathcal{F})_{\mid \mathcal{N}_{v}}$, which gives the $\swarrow$-orientation of $\mathcal{N}_{v}$ in $\pi(\mathcal{F})$ on the left hand side, and a example that illustrates a not well-oriented gadget $\mathcal{N}$ in the example partition $\mathcal{H}^{(*)}$-partition of $\mathcal{N}$ on the right hand side.

$\left|\pi^{\prime}\right| \leq|\pi|, \mathcal{N}_{u}$ is well-oriented in $\pi^{\prime}$, and also preserves the orientation of $\mathcal{N}_{w}$ for $w \neq$ $u, w \in \mathbb{V}$ (i.e. $\mathcal{N}_{w}$ is well-oriented in $\pi$ if and only if $\mathcal{N}_{w}$ is well-oriented in $\pi^{\prime}$ ).

Proof. Let $\pi, u$ fulfil the assumptions. Our proof starts with the observation that vertices of $\mathcal{P}(\mathcal{G}, e)_{\mid \pi, u}$ can be divided into three types: elements of $\mathcal{V}_{u}$, elements which can be represented in the form $\langle u, l\rangle$ for some $l \in N_{\mathcal{G}}^{+}(u)$, and elements which can be represented in the form $\langle r, u\rangle$ for some $r \in N_{\mathcal{G}}^{-}(u)$. Let $L:=\left\{\langle u, l\rangle:\langle u, l\rangle \in \mathbb{V}_{\mathcal{G} \mid \pi, u}\right\}, R:=\left\{\langle r, u\rangle:\langle r, u\rangle \in \mathbb{V}_{\mathcal{G} \mid \pi, u}\right\}$ and suppose that $L=\left\{\left\langle u, l_{1}\right\rangle,\left\langle u, l_{2}\right\rangle, \ldots,\left\langle u, l_{i}\right\rangle\right\}, R=\left\{\left\langle r_{1}, u\right\rangle,\left\langle r_{2}, u\right\rangle, \ldots,\left\langle r_{j}, u\right\rangle\right\}$, where $e\left(\left\langle u, l_{1}\right\rangle\right)<e\left(\left\langle u, l_{2}\right\rangle\right)<\ldots<e\left(\left\langle u, l_{i}\right\rangle\right)$, and $e\left(\left\langle r_{1}, u\right\rangle\right)<e\left(\left\langle r_{2}, u\right\rangle\right)<\ldots<e\left(\left\langle r_{j}, u\right\rangle\right)$. Since $\mathcal{G}$ is without self-loops, we infer that $\left\{l_{1}, l_{2}, \ldots, l_{i}\right\} \cap\left\{r_{1}, r_{2}, \ldots, r_{j}\right\}=\emptyset$. We also denote by $\mathfrak{h}_{\mid u}^{\pi}(P)$ the subsequence of $\mathfrak{h}^{\pi}(P)$ which is an $\mathbb{E}_{\mathcal{G}}$-path given by $\mathcal{V}\left(\mathfrak{h}^{\pi}(P)\right) \cap \mathcal{N}_{u}$, since $\mathcal{V}\left(\mathfrak{h}^{\pi}(P)\right) \backslash \mathcal{N}_{u}$ can contain at most sources in $\mathcal{P}(\mathcal{G}, e)$ if $\mathcal{V}\left(\mathfrak{h}^{\pi}(P)\right) \cap \mathcal{N}_{u} \neq \emptyset$, where $P \in \pi$. Additionally, we denote by $\mathfrak{h}_{\mid u}^{\pi}(v)$ the path $\mathfrak{h}_{\mid u}^{\pi}(P)$, where $v \in P$.

Step 1. We show that $\left|\pi_{\mid u}\right| \geq|\mathbb{E}|+1+\min \{i, j\}$. To do this, suppose first that for every integer $k$ that satisfies $1 \leq k \leq|\mathbb{E}|$ there exists $P \in \pi_{\mid u}$ for which $\mathfrak{h}_{\mid u}^{\pi}(P)$ is a nonempty subsequence of $\swarrow_{u}^{k}$. Thus the partition $\pi$ has at least $|\mathbb{E}|$ elements, i.e. the ones forming the subsequence of $\swarrow_{u}^{k}$ for $1 \leq k \leq|\mathbb{E}|$. These elements are also different from any $\mathcal{V}\left(\mathfrak{h}^{\pi}(v)\right)$ where $v \in R \cup\left\{u_{0,0}\right\}$, since $\mathcal{V}\left(\swarrow_{u}^{k}\right) \cap\left(R \cup\left\{u_{0,0}\right\}\right)=\emptyset$ for each $k$. In addition, $\mathcal{V}\left(\mathfrak{h}^{\pi}\left(v_{1}\right)\right) \neq \mathcal{V}\left(\mathfrak{h}^{\pi}\left(v_{2}\right)\right)$ for different $v_{1}, v_{2} \in R \cup\left\{u_{0,0}\right\}$, as $v_{1}, v_{2}$ have no incoming arcs in $\mathcal{P}(\mathcal{G}, e)_{\mid \pi, u}$. Thus finally $\left|\pi_{\mid u}\right| \geq|\mathbb{E}|+\left|R \cup\left\{u_{0,0}\right\}\right|=|\mathbb{E}|+1+j \geq|\mathbb{E}|+1+\min \{i, j\}$.

Suppose now that there exists $k$ that satisfies $1 \leq k \leq|\mathbb{E}|$, for which there is no $P \in \pi_{\mid u}$ such that the whole sequence $\mathfrak{h}_{\mid u}^{\pi}(P)$ is a subsequence of $\swarrow_{u}^{k}$. From Lemma 5.2 we have that for each $1 \leq d \leq|\mathbb{E}|, \mathfrak{h}^{\pi}\left(u_{k, d}\right)$ is a subsequence of $\swarrow_{u}^{k}$ or $\searrow_{u}^{d}$, hence by assumption $\mathcal{V}\left(\mathfrak{h}^{\pi}\left(u_{k, d}\right)\right) \subseteq \mathcal{V}\left(\searrow_{u}^{d}\right)$ for each $d$. Thus $\mathfrak{h}^{\pi}\left(u_{k, 1}\right), \mathfrak{h}^{\pi}\left(u_{k, 2}\right), \ldots, \mathfrak{h}^{\pi}\left(u_{k,|\mathbb{E}|}\right)$ are pairwise different and $\mathfrak{h}^{\pi}\left(u_{k, d}\right) \neq \mathfrak{h}^{\pi}(v)$ for every $1 \leq d \leq|\mathbb{E}|, v \in L \cup\left\{u_{0,0}\right\}$ (note that the possibility $\mathfrak{h}^{\pi}\left(u_{k, 0}\right)=\mathfrak{h}^{\pi}(v)$ for some $v \in L \cup\left\{u_{0,0}\right\}$ is not excluded). Additionally, $\mathfrak{h}^{\pi}\left(v_{1}\right) \neq \mathfrak{h}^{\pi}\left(v_{2}\right)$ for different $v_{1}, v_{2} \in L \cup\left\{u_{0,0}\right\}$ as $v_{1}, v_{2}$ have no incoming arcs, hence $\left|\pi_{\mid u}\right| \geq|\mathbb{E}|+\left|L \cup\left\{u_{0,0}\right\}\right|=|\mathbb{E}|+1+i \geq|\mathbb{E}|+1+\min \{i, j\}$ and the proof of this step is complete.

Step 2. We describe here a property of arcs in $\mathcal{G}(\mathcal{P}(\mathcal{G}, e), \pi)$ whose both tail and head cross different gadgets. Let $\left\langle P_{1}, P_{2}\right\rangle$ be an arc of $\mathcal{G}(\mathcal{P}(\mathcal{G}, e), \pi)$ such that $P_{1} \cap \mathcal{V}_{v} \neq \emptyset$, 

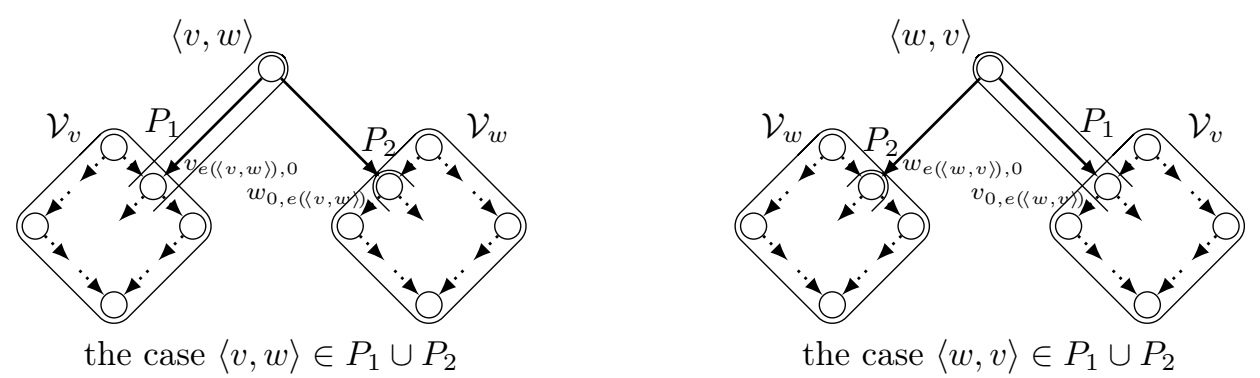

Figure 10: An illustration of the construction from the proof of Lemma [5.8, step 2.

$P_{2} \cap \mathcal{V}_{w} \neq \emptyset$, and $v \neq w$, where $v, w \in \mathbb{V}$. We show that it is possible only in two cases, depending on the direction of the arc between $v$ and $w$ :

(i) $\langle v, w\rangle, v_{e}(\langle v, w\rangle), 0 \in P_{1}, w_{0, e(\langle v, w\rangle)} \in P_{2}$,

(ii) or $\langle w, v\rangle, v_{0, e}(\langle w, v\rangle) \in P_{1}, w_{e}(\langle w, v\rangle), 0 \in P_{2}$.

Observe that since $\mathcal{V}_{v} \underset{\mathbb{E}_{\mathcal{G}}}{\curvearrowright} \mathcal{V}_{w}=\emptyset$ and $P_{1} \underset{\mathbb{E}_{\mathcal{G}}}{\curvearrowright} P_{2} \neq \emptyset$, one of vertices in the arc that connects $P_{1}$ with $P_{2}$ must be outside of $\mathcal{V}_{v}, \mathcal{V}_{w}$ (see Fig. 10). This vertex has to be a part of a Hamiltonian path intersecting the sets $\mathcal{V}_{v}, \mathcal{V}_{w}$, hence it has the form $\langle v, x\rangle,\langle w, x\rangle,\langle x, v\rangle$, or $\langle x, w\rangle$. The only vertices of the form that can be connected to both $\mathcal{V}_{v}$ and $\mathcal{V}_{w}$ are $\langle v, w\rangle$ and $\langle w, v\rangle$. Therefore, we have $\langle v, w\rangle \in P_{1} \cup P_{2}$ or $\langle w, v\rangle \in P_{1} \cup P_{2}$. We show that $(i)$ holds for $\langle v, w\rangle \in P_{1} \cup P_{2}$ and (ii) in the second case. We prove only the first case, the second case is analogous. Let us assume that $\langle v, w\rangle \in P_{1} \cup P_{2}$ and suppose first, contrary to our claim that $\langle v, w\rangle \notin P_{1}$. Then $\langle v, w\rangle \in P_{2}$. Obviously, $\langle v, w\rangle$ is adjacent only with $v_{e}(\langle v, w\rangle), 0, w_{0, e}(\langle v, w\rangle)$ and both cannot belong at the same time to $P_{2}$, since $\langle v, w\rangle$ is a source in $\mathcal{P}(\mathcal{G}, e)$ and $P_{2}$ determines the path $\mathfrak{h}^{\pi}(\langle v, w\rangle)$. Note, that an arbitrary directed path of $\mathcal{P}(\mathcal{G}, e)$ can go through at most one vertex outside of the gadgets of the form (5.2). Additionally, there are no arcs that connect vertices belonging to two different gadgets. Hence $P_{2} \backslash\{\langle v, w\rangle\} \subseteq \mathcal{V}_{w}$ since $\langle v, w\rangle \in P_{2}$ and $P_{2} \cap \mathcal{V}_{w} \neq \emptyset$. Consequently, $\langle v, w\rangle$ is the only vertex that belongs to $P_{2}$ and is adjacent to any vertex of $\mathcal{V}_{v}$. Then, $\left\langle\langle v, w\rangle, v_{e}(\langle v, w\rangle), 0\right\rangle \in P_{2} \underset{\mathbb{E}_{\mathcal{G}}}{\curvearrowright} P_{1}$ and finally, $\left\langle P_{2}, P_{1}\right\rangle$ is an arc of $\mathcal{G}(\mathcal{P}(\mathcal{G}, e), \pi)$, but this contradicts the assumption that $\mathcal{G}(\mathcal{P}(\mathcal{G}, e), \pi)$ is acyclic. Since $\langle v, w\rangle \in P_{1}$, it is easy to check that $v_{e(\langle v, w\rangle), 0} \in P_{1}, w_{0, e(\langle v, w\rangle)} \in P_{2}$.

Step 3. We prove here the final conclusion. Let us consider two $\mathcal{H}^{(*)}$-partitions of $\mathcal{P}(\mathcal{G}, e)_{\mid \pi, u}$

$$
\begin{aligned}
& \widehat{\mathcal{L}}_{u}:=\left\{\mathcal{V}\left(\swarrow_{u}^{n}\right): 0 \leq n \leq|\mathbb{E}| \wedge n \notin e(L)\right\} \cup \\
& \left.\left\{\mathcal{V}\left(\left\langle u, l_{n}\right\rangle\right\rangle \frown \swarrow_{u}^{e\left(\left\langle u, l_{n}\right\rangle\right)}\right): 1 \leq n \leq i\right\} \cup\left\{\left\{\left\langle r_{n}, u\right\rangle\right\}: 1 \leq n \leq j\right\}, \\
& \widehat{\mathcal{R}}_{u}:=\left\{\mathcal{V}\left(\searrow_{u}^{n}\right): 0 \leq n \leq|\mathbb{E}| \wedge n \notin e(R)\right\} \cup \\
& \left\{\mathcal{V}\left(\left\langle\left\langle r_{n}, u\right\rangle\right\rangle \frown \searrow^{e\left(\left\langle r_{n}, u\right\rangle\right)}\right): 1 \leq n \leq j\right\} \cup\left\{\left\{\left\langle u, l_{n}\right\rangle\right\}: 1 \leq n \leq i\right\} .
\end{aligned}
$$

It is clear that $\left|\widehat{\mathcal{L}}_{u}\right|=|\mathbb{E}|+1+j,\left|\widehat{\mathcal{R}}_{u}\right|=|\mathbb{E}|+1+i$. From the assumption about $\mathcal{G}$ we obtain that in-degree or out-degree of $u$ is 1 . Let us assume that in-degree of $u$ is 1 , thus there exists $\mathfrak{r}$ such that $N_{\mathcal{G}}^{-}(u)=\{\mathfrak{r}\}$ (the second case $\left|N_{\mathcal{G}}^{+}(u)\right|=1$ is analogous). The situation that $\langle\mathfrak{r}, u\rangle \notin \mathbb{V}_{\mathcal{G} \mid \pi, u}$ is not excluded here, hence $j \leq 1$. We split the proof into the following cases: 


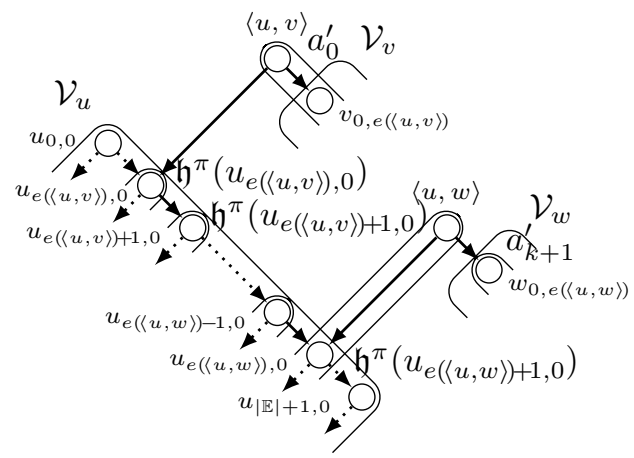

Figure 11: An illustration of the construction from the proof of Lemma [5.8, step 3, case 1.

1. Suppose that $j=1, i \geq 1$. From Step 1. we have that $\left|\pi_{\mid u}\right| \geq|\mathbb{E}|+2=\left|\widehat{\mathcal{L}}_{u}\right|$, and consequently $\left|\pi^{\prime}\right| \leq|\pi|$ where $\pi^{\prime}$ is defined by $\pi^{\prime}:=\left(\pi \backslash \pi_{\mid u}\right) \cup \widehat{\mathcal{L}}_{u}$. Obviously, $\pi^{\prime}$ is an $\mathcal{H}^{*}$-partition of $\mathcal{P}(\mathcal{G}, e)$ and $\pi^{\prime}$ fulfils the condition about well-ordering of gadgets. To complete the proof it is enough to show that $\mathcal{G}\left(\mathcal{P}(\mathcal{G}, e), \pi^{\prime}\right)$ is acyclic. Suppose, contrary to our claim, that there exists a cycle $\mathfrak{a}$ in $\mathcal{G}\left(\mathcal{P}(\mathcal{G}, e), \pi^{\prime}\right)$. As $\mathcal{G}(\mathcal{P}(\mathcal{G}, e), \pi)$ is acyclic we have that $\mathfrak{a}$ has to cross vertices of $\widehat{\mathcal{L}}_{u}$. To obtain a contradiction, we show that it is possible to replace every maximal subpath of $\mathfrak{a}$ that consists of vertices from $\widehat{\mathcal{L}}_{u}$ by a path which is obtained from vertices of $\pi_{\mid u}$ in such a way that the modified path $\mathfrak{a}^{\prime}$ is a cycle. By repetitive application of the procedure we obtain a cycle in $\mathcal{G}(\mathcal{P}(\mathcal{G}, e), \pi)$.

Let us take such a maximal subpath $\mathfrak{a}^{\prime}=\left\langle a_{1}^{\prime}, a_{2}^{\prime}, \ldots, a_{k}^{\prime}\right\rangle$ of $\mathfrak{a}$ with elements of $\widehat{\mathcal{L}}_{u}$ and denote by $a_{0}^{\prime}, a_{k+1}^{\prime}$ vertices that belong to $\mathfrak{a}$ and $\left\langle a_{0}^{\prime}, a_{1}^{\prime}\right\rangle,\left\langle a_{k}^{\prime}, a_{k+1}^{\prime}\right\rangle$ are arcs of $\mathcal{G}\left(\mathcal{P}(\mathcal{G}, e), \pi^{\prime}\right)$. We have that $\mathfrak{a} \neq \mathfrak{a}^{\prime}$ and $a_{0}^{\prime}, a_{k+1}^{\prime} \neq a_{1}^{\prime}, a_{2}^{\prime}, \ldots, a_{k}^{\prime}$ since $\widehat{\mathcal{L}}_{u}$ is an $\mathcal{H}^{(*)_{-}}$ partitions of $\mathcal{P}(\mathcal{G}, e)_{\mid \pi, u}$. Since $\{\langle\mathfrak{r}, u\rangle\}$ is a source in $\mathcal{G}\left(\mathcal{P}(\mathcal{G}, e), \pi^{\prime}\right)$ it cannot occur in a cycle, therefore for each $i=1,2, \ldots, k$ the property $a_{i}^{\prime} \cap \mathcal{V}_{u} \neq \emptyset$ holds. For similar reasons, the edges in $\mathbb{E}$ that witness $\left\langle a_{0}^{\prime}, a_{1}^{\prime}\right\rangle,\left\langle a_{k}^{\prime}, a_{k+1}^{\prime}\right\rangle$ have the form $\langle u, v\rangle,\langle u, w\rangle \in \mathbb{E}$ where $a_{0}^{\prime} \cap \mathcal{V}_{v} \neq \emptyset, a_{k+1}^{\prime} \cap \mathcal{V}_{w} \neq \emptyset$. Then from Step 2. we have that $\langle u, v\rangle \in a_{0}^{\prime}, u_{e}(\langle u, v\rangle), 0 \in a_{1}^{\prime}$, $\langle u, w\rangle, u_{e}(\langle u, w\rangle), 0 \in a_{k}^{\prime}, w_{0, e(\langle u, w\rangle)} \in a_{k+1}^{\prime}$ and, in consequence, $v \in L, w \in \mathcal{N}_{\mathcal{G}}^{+}(u) \backslash L$. Additionally, $e(\langle u, v\rangle)<e(\langle u, w\rangle)$ (see Fig. 11). Let us consider a sequence

$$
\mathfrak{b}=\left\langle\mathfrak{h}^{\pi}\left(u_{e}(\langle u, v\rangle), 0\right), \mathfrak{h}^{\pi}\left(u_{e(\langle u, v\rangle)+1,0}\right), \mathfrak{h}^{\pi}\left(u_{e(\langle u, v\rangle)+2,0}\right), \ldots, \mathfrak{h}^{\pi}\left(u_{e}(\langle u, w\rangle), 0\right)\right\rangle
$$

of vertices of $\pi_{\mid u}$. Obviously, vertices of this sequence do not have to be pairwise different. Therefore let us consider the maximal subsequence $\mathfrak{b}^{\prime}=\left\langle b_{1}, b_{2}, \ldots, b_{k^{\prime}}\right\rangle$ of $\mathfrak{b}$ such that $b_{i} \neq b_{i+1}$ for $i=1,2, \ldots, k^{\prime}-1$. It is easy to check that for each $i$ there exists $j$ such that $e(\langle u, v\rangle) \leq j<e(\langle u, w\rangle), b_{i}=\mathfrak{h}^{\pi}\left(u_{j, 0}\right), b_{i+1}=\mathfrak{h}^{\pi}\left(u_{j+1,0}\right)$, hence $\left\langle b_{i}, b_{i+1}\right\rangle$ is an arc of $\mathcal{G}(\mathcal{P}(\mathcal{G}, e), \pi)$, since $\left\langle u_{j, 0}, u_{j+1,0}\right\rangle \in \mathbb{E}_{\mathcal{G}}$, and finally $\mathfrak{b}^{\prime}$ is a path of $\mathcal{G}(\mathcal{P}(\mathcal{G}, e), \pi)$. Additionally, $\left\langle a_{0}^{\prime}, b_{1}\right\rangle,\left\langle b_{k^{\prime}}, a_{k+1}^{\prime}\right\rangle$ are arcs of $\mathcal{G}(\mathcal{P}(\mathcal{G}, e), \pi)$, hence we obtain that the subpath $\mathfrak{a}^{\prime}$ can be replaced by $\mathfrak{b}^{\prime}$.

2. Suppose that $j=i=0$. From Step 1. we have that $\left|\pi_{\mid u}\right| \geq|\mathbb{E}|+1=\left|\widehat{\mathcal{L}}_{u}\right|$. Then the proof completes if we take $\pi^{\prime}:=\left(\pi \backslash \pi_{\mid u}\right) \cup \widehat{\mathcal{L}}_{u}$, since every arc of $\mathcal{G}\left(\mathcal{P}(\mathcal{G}, e), \pi^{\prime}\right)$ that joins vertices of $\pi \backslash \pi_{\mid u}$ and $\widehat{\mathcal{L}}_{u}$ is directed towards $\widehat{\mathcal{L}}_{u}$. 
3. Suppose that $j=0, i \geq 1$. From Step 1. we have that $\left|\pi_{\mid u}\right| \geq|\mathbb{E}|+1=\left|\widehat{\mathcal{L}}_{u}\right|$. Let $\pi^{\prime}:=\left(\pi \backslash \pi_{\mid u}\right) \cup \widehat{\mathcal{L}}_{u}$. Obviously, $\left|\pi^{\prime}\right| \leq|\pi|$ and $\pi^{\prime}$ is an $\mathcal{H}^{*}$-partition of $\mathcal{P}(\mathcal{G}, e)$ that fulfils the condition about well-ordering of gadgets. It is left to show that $\mathcal{G}\left(\mathcal{P}(\mathcal{G}, e), \pi^{\prime}\right)$ is acyclic. Suppose, contrary to our claim, that there exists a cycle $\mathfrak{a}$ of $\mathcal{G}\left(\mathcal{P}(\mathcal{G}, e), \pi^{\prime}\right)$. Similar to the first case, we infer that $\mathfrak{a}$ has to cross vertices of $\widehat{\mathcal{L}}_{u}$, but since $j=0$, $\{\langle\mathfrak{r}, u\rangle\}$ need not be a source in $\mathcal{G}\left(\mathcal{P}(\mathcal{G}, e), \pi^{\prime}\right)$, and $\mathfrak{a}$ can cross $\widehat{\mathcal{L}}_{u}$, not only by going in and going out from the "right hand side" of $\mathcal{N}_{u}$ (as in Fig. 11), but also by going in from the "left hand side" of $\mathcal{N}_{u}$ traversing the vertex $P=\mathcal{V}\left(\mathfrak{h}^{\pi^{\prime}}(\langle\mathfrak{r}, u\rangle)\right)$. In the case, when there exists a cycle of $\mathcal{G}\left(\mathcal{P}(\mathcal{G}, e), \pi^{\prime}\right)$ that goes to $\widehat{\mathcal{L}}_{u}$ traversing $P$, we prove that $\left|\pi^{\prime}\right|<|\pi|$. Then we conclude that $\pi^{\prime \prime}=\left(\pi^{\prime} \backslash\{P\}\right) \cup\{P \backslash\{\langle\mathfrak{r}, u\rangle\},\{\langle\mathfrak{r}, u\rangle\}\}$, which is an $\mathcal{H}^{*}$-partition of $\mathcal{P}(\mathcal{G}, e)$ that is created by dividing $P$ into two parts $P \backslash\{\langle\mathfrak{r}, u\rangle\},\{\langle\mathfrak{r}, u\rangle\}$, has at most $|\pi|$ elements and preserves the orientanion of gadgets. Additionally, $\{\langle\mathfrak{r}, u\rangle\}$ is a source in $\mathcal{G}\left(\mathcal{P}(\mathcal{G}, e), \pi^{\prime \prime}\right)$. Now, every cycle has to cross vertices of $\widehat{\mathcal{L}}_{u}$, go in and go out from the "right hand side" of $\mathcal{N}_{u}$. The application of the proof idea of the first case completes the proof.

Let us take a cycle of $\mathcal{G}\left(\mathcal{P}(\mathcal{G}, e), \pi^{\prime}\right)$ that goes into $\widehat{\mathcal{L}}_{u}$ traversing $P$. Now we prove that $\left|\pi^{\prime}\right|<|\pi|$. For this purpose it is enough to show that $\left|\pi_{\mid u}\right|>|\mathbb{E}|+1=\left|\widehat{\mathcal{L}}_{u}\right|$. Suppose, contrary to our claim, that $\left|\pi_{\mid u}\right| \leq|\mathbb{E}|+1$. From Lemma 5.4 we conclude that $|\mathbb{E}|+1 \leq\left|\pi_{\mid \mathcal{N}_{u}}\right| \leq\left|\pi_{\mid u}\right| \leq|\mathbb{E}|+1$ and in addition $\pi_{\mid \mathcal{N}_{u}}=\mathcal{L}_{u}$ or $\pi_{\mid \mathcal{N}_{u}}=\mathcal{R}_{u}$. Note in this case also $\left|\pi_{\left|\mathcal{N}_{u}\right|}\right|=\left|\pi_{\mid u}\right|$. Thus, when $\pi_{\mid \mathcal{N}_{u}}=\mathcal{L}_{u}$ we get equalities $\pi_{\mid u}=\widehat{\mathcal{L}}_{u}, \pi^{\prime}=\pi$, but this contradicts our assumption that $\mathcal{G}(\mathcal{P}(\mathcal{G}, e), \pi)$ is acyclic. In consequence, $\pi_{\mid \mathcal{N}_{u}}=\mathcal{R}_{u}$. Let us focus on the vertex $\left\langle u, l_{1}\right\rangle \in \mathbb{V}_{\mathcal{G} \mid \pi, u}$. This vertex is adjacent only with $u_{e}\left(\left\langle u, l_{1}\right\rangle\right), 0$ from $\mathbb{V}_{\mathcal{G} \mid \pi, u}$, which also belongs to $\mathcal{V}\left(\searrow_{u}^{0}\right)$. Hence there exists $Q \in \pi_{\mid u}$ that contains $\left\{\left\langle u, l_{1}\right\rangle\right\} \cup \mathcal{V}\left(\searrow_{u}^{0}\right)$, since $\left|\pi_{\mid u}\right|=\left|\mathcal{R}_{u}\right|$. However $\left\{\left\langle u, l_{1}\right\rangle\right\} \cup \mathcal{V}\left(\searrow_{u}^{0}\right)$ contains two sources in $\mathcal{P}(\mathcal{G}, e)$, namely $\left\langle u, l_{1}\right\rangle$ and $u_{0,0}$. This is in contradiction with the fact that all the vertices of $Q$ are in a single Hamiltonian path.

4. Suppose that $j=1, i=0$. From Step 1. we have that $\left|\pi_{\mid u}\right| \geq|\mathbb{E}|+1=\left|\widehat{\mathcal{R}}_{u}\right|$. Let $\pi^{\prime}:=\left(\pi \backslash \pi_{\mid u}\right) \cup \widehat{\mathcal{R}}_{u}$. Obviously, $\left|\pi^{\prime}\right| \leq|\pi|$ and $\pi^{\prime}$ is an $\mathcal{H}^{*}$-partition of $\mathcal{P}(\mathcal{G}, e)$ that fulfils the condition about well-ordering of gadgets. We show that $\mathcal{G}\left(\mathcal{P}(\mathcal{G}, e), \pi^{\prime}\right)$ is acyclic. Suppose, contrary to our claim, that there exists a cycle $\mathfrak{a}$ of $\mathcal{G}\left(\mathcal{P}(\mathcal{G}, e), \pi^{\prime}\right)$. It is easy to check that $\mathfrak{a}$ has to go into $\widehat{\mathcal{L}}_{u}$ from the "left hand side" of $\mathcal{N}_{u}$ and go out through the vertex $P=\mathcal{V}\left(\mathfrak{h}^{\pi^{\prime}}(\langle\mathfrak{r}, u\rangle)\right)$ - on the "right hand side". In such a case we show, similarly to the third case that $\left|\pi_{\mid u}\right|>|\mathbb{E}|+1$ and then we conclude that $\pi^{\prime \prime}=\left(\pi^{\prime} \backslash\{P\}\right) \cup\{P \backslash\{\langle\mathfrak{r}, u\rangle\},\{\langle\mathfrak{r}, u\rangle\}\}$ is an $\mathcal{H}^{*}$-partition of $\mathcal{P}(\mathcal{G}, e)$ that has at most $|\pi|$ elements, preserve the orientation of gadgets, and $\{\langle\mathfrak{r}, u\rangle\}$ is a source in $\mathcal{G}\left(\mathcal{P}(\mathcal{G}, e), \pi^{\prime \prime}\right)$. Since we can modify $\pi$ in such a way that every cycle cannot go out from $\widehat{\mathcal{R}}_{u}$, the proof is completed.

Let us take a cycle of $\mathcal{G}\left(\mathcal{P}(\mathcal{G}, e), \pi^{\prime}\right)$ that goes out from $\widehat{\mathcal{R}}_{u}$ through $P$, and suppose, contrary to our claim, that $\left|\pi_{\mid u}\right| \leq|\mathbb{E}|+1$. From Lemma 5.4 we conclude that $|\mathbb{E}|+1 \leq$ $\left|\pi_{\mid \mathcal{N}_{u}}\right| \leq\left|\pi_{\mid u}\right| \leq|\mathbb{E}|+1$ and in addition $\pi_{\mid \mathcal{N}_{u}}=\mathcal{L}_{u}$ or $\pi_{\mid \mathcal{N}_{u}}=\mathcal{R}_{u}$. Note that since $\left|\pi_{\mid \mathcal{N}_{u}}\right|=\left|\pi_{\mid u}\right|$ in the case $\pi_{\mid \mathcal{N}_{u}}=\mathcal{R}_{u}$ we get equalities $\pi_{\mid u}=\widehat{\mathcal{R}}_{u}, \pi^{\prime}=\pi$, but this contradicts our assumption that $\mathcal{G}(\mathcal{P}(\mathcal{G}, e), \pi)$ is acyclic. In consequence, $\pi_{\mid \mathcal{N}_{u}}=\mathcal{L}_{u}$. Let us focus on the vertex $\langle\mathfrak{r}, u\rangle \in \mathbb{V}_{\mathcal{G} \mid \pi, u}$. This vertex is adjacent only with $u_{0, e(\langle\mathfrak{r}, u\rangle)}$ from $\mathbb{V}_{\mathcal{G} \mid \pi, u}$, which also belongs to $\mathcal{V}\left(\swarrow_{u}^{0}\right)$. Hence there exists $Q \in \pi_{\mid u}$ that contains $\{\langle\mathfrak{r}, u\rangle\} \cup \mathcal{V}\left(\swarrow_{u}^{0}\right)$, since $\left|\pi_{\mid u}\right|=\left|\mathcal{L}_{u}\right|$. However $\{\langle\mathfrak{r}, u\rangle\} \cup \mathcal{V}\left(\swarrow_{u}^{0}\right)$ contains two sources in 
$\mathcal{P}(\mathcal{G}, e)$, namely $\langle\mathfrak{r}, u\rangle$ and $u_{0,0}$. This is in contradiction with the fact that all the vertices of $Q$ are in a single Hamiltonian path.

Lemma 5.9. Let $\pi$ be an $\mathcal{H}^{(*)}$-partition of $\mathcal{P}(\mathcal{G}, e)$ for which all gadgets are well-oriented. Then $\{\langle v, u\rangle:\{\langle v, u\rangle\} \in \pi\}$ is a feedback arc set which has size at most $|\pi|-|\mathbb{V}| \cdot(|\mathbb{E}|+1)$.

Proof. Let $\pi$ fulfil the assumptions and introduce $\mathcal{F}:=\{\langle v, u\rangle:\{\langle v, u\rangle\} \in \pi\}, \mathcal{O}:=$ $\{\{\langle v, u\rangle\} \in \pi\}$. Suppose, contrary to our claim, that $\mathcal{F}$ is not a feedback arc set of $\mathcal{G}$. Then there exists $\mathbb{E} \backslash \mathcal{F}$-path $\mathfrak{c}:=\left\langle c_{1}, c_{2}, \ldots, c_{k}\right\rangle$ of $\mathcal{G}$ such that $k>2$ and $c_{1}=c_{k}$. Additionally, we let $c_{k+1}:=c_{2}$ and we use the following notation:

$$
\begin{aligned}
& \swarrow^{\langle v, u\rangle}:=\langle\langle v, u\rangle\rangle \frown \swarrow_{v}^{e(\langle v, u\rangle),}, \\
& \langle v, u\rangle \searrow:=\langle\langle v, u\rangle\rangle \frown \searrow_{v u}^{e(\langle v, u\rangle)},
\end{aligned}
$$

where $\langle v, u\rangle \in \mathbb{E}$. It is easy to check that for every $i$ which fulfils $1 \leq i<k$, exactly one of two conditions $\mathfrak{h}^{\pi}\left(\left\langle c_{i}, c_{i+1}\right\rangle\right)=\swarrow^{\left\langle c_{i}, c_{i+1}\right\rangle}, \mathfrak{h}^{\pi}\left(\left\langle c_{i}, c_{i+1}\right\rangle\right)=\left\langle c_{i}, c_{i+1}\right\rangle \searrow$ is satisfied, since all gadgets are well-oriented. Moreover from the fact that no gadget has both orientations in $\pi$, we conclude that $\mathfrak{h}^{\pi}\left(\left\langle c_{i}, c_{i+1}\right\rangle\right)={ }^{\left\langle c_{i}, c_{i+1}\right\rangle} \searrow$ if and only if $\mathfrak{h}^{\pi}\left(\left\langle c_{i+1}, c_{i+2}\right\rangle\right) \neq \swarrow^{\left\langle c_{i+1}, c_{i+2}\right\rangle}$, for $i=1,2, \ldots, k-1$. In consequence, every gadget $\mathcal{N}_{c_{i}}$ is $\swarrow$-oriented or every gadget $\mathcal{N}_{c_{i}}$ is \-oriented, therefore one of the following sequences

$$
\begin{aligned}
& \left\langle\swarrow_{c_{1}}^{0}, \mathfrak{h}^{\pi}\left(\left(c_{1}\right)_{1,0}\right), \ldots, \mathfrak{h}^{\pi}\left(\left(c_{1}\right)_{e}\left(\left\langle c_{1}, c_{2}\right\rangle\right)-1,0\right), \swarrow^{\left\langle c_{1}, c_{2}\right\rangle},\right. \\
& \swarrow_{c_{2}}^{0}, \mathfrak{h}^{\pi}\left(\left(c_{2}\right)_{1,0}\right), \ldots, \mathfrak{h}^{\pi}\left(\left(c_{1}\right)_{e}\left(\left\langle c_{2}, c_{3}\right\rangle\right)-1,0\right), \swarrow^{\left\langle c_{2}, c_{3}\right\rangle}, \\
& \left.\ldots, \swarrow_{c_{k-1}}^{0}, \mathfrak{h}^{\pi}\left(\left(c_{k-1}\right)_{1,0}\right), \ldots, \mathfrak{h}^{\pi}\left(\left(c_{k-1}\right)_{e\left(\left\langle c_{k-1}, c_{k}\right\rangle\right)-1,0}\right), \swarrow^{\left\langle c_{k-1}, c_{k}\right\rangle}, \swarrow_{c_{1}}^{0}\right\rangle, \\
& \left\langle\searrow_{c_{k}}^{0}, \mathfrak{h}^{\pi}\left(\left(c_{k}\right)_{0,1}\right), \ldots, \mathfrak{h}^{\pi}\left(\left(c_{k}\right)_{0, e\left(\left\langle c_{k-1}, c_{k}\right\rangle\right)-1}\right),{ }^{\left\langle c_{k-1}, c_{k}\right\rangle} \searrow,\right. \\
& \searrow_{c_{k-1}}^{0}, \mathfrak{h}^{\pi}\left(\left(c_{k-1}\right)_{0,1}\right), \ldots, \mathfrak{h}^{\pi}\left(( c _ { k - 1 } ) _ { 0 , e } \left(\left\langle c_{\left.\left.c_{k-2}, c_{k-1}\right\rangle\right)-1}\right),{ }^{\left\langle c_{k-2}, c_{k-1}\right\rangle} \searrow,\right.\right. \\
& \left.\ldots, \searrow_{c_{2}}^{0}, \mathfrak{h}^{\pi}\left(\left(c_{2}\right)_{0,1}\right), \ldots, \mathfrak{h}^{\pi}\left(\left(c_{2}\right)_{0, e\left(\left\langle c_{1}, c_{2}\right\rangle\right)-1}\right),{ }^{\left\langle c_{1}, c_{2}\right\rangle} \searrow, \searrow_{c_{k}}^{0}\right\rangle
\end{aligned}
$$

represents a cycle of the $\mathrm{DAG} \mathcal{G}(\mathcal{P}(\mathcal{G}, e), \pi)$, but $\mathcal{G}(\mathcal{P}(\mathcal{G}, e), \pi)$ is acyclic since $\pi$ is an $\mathcal{H}^{(*)}$-partition of $\mathcal{P}(\mathcal{G}, e)$, a contradiction. This finishes the proof, that $\mathcal{F}$ is a a feedback arc set of $\mathcal{G}$.

It remains to show that $\mathcal{F} \leq|\pi|-|\mathbb{V}| \cdot(|\mathbb{E}|+1)$, but it is obvious since $\pi \backslash \mathcal{O}=\bigcup_{v \in \mathbb{V}} \pi_{\mid v}$; $\left|\pi_{\mid v}\right|=|\mathbb{E}|+1$, for every $v \in \mathbb{V}$; and $\pi_{\mid v_{1}}, \pi_{\mid v_{2}}$ are disjoint for every different $v_{1}, v_{2} \in \mathbb{V}$.

Theorem 5.10. The FAS problem is reducible to the AHP problem.

Proof. For each instance $\mathcal{G}=\langle\mathbb{V}, \mathbb{E}\rangle, k$ of FAS we can construct $\mathcal{P}(\mathcal{G}, e)$, a number $k+$ $|\mathbb{V}| \cdot(|\mathbb{E}|+1)$ and consider them to be an instance of AHP. This translation can clearly be done in logspace. By Lemma 5.6 if $\mathcal{G}$ has a feedback arc set of size not greater than $k$, then $\mathcal{P}(\mathcal{G}, e)$ has a $\mathcal{H}^{(*)}$ partition of size not greater than $k+|\mathbb{V}| \cdot(|\mathbb{E}|+1)$. By Lemma 5.8 composed with Lemma 5.9 we obtain that when $\mathcal{P}(\mathcal{G}, e)$ has a $\mathcal{H}^{(*)}$ partition of size not greater than $k+|\mathbb{V}| \cdot(|\mathbb{E}|+1)$ then $\mathcal{G}$ has a feedback arc set of size not greater than $k$. Therefore translation $\mathcal{G}, k$ to $\mathcal{P}(\mathcal{G}, e), k+|\mathbb{V}| \cdot(|\mathbb{E}|+1)$ is indeed a reduction. 
According to the above theorem, AHP is NP-complete. Additionally, vertices of the constructed digraph $\mathcal{P}(\mathcal{G}, e)$ have restricted in-degree and out-degree by 2 . Hence we finally have the following theorem.

Theorem 5.11. The AHP problem is NP-complete for digraphs in which no vertex has in-degree or out-degree greater than 2.

\section{Constructive abstract proof graphs}

In Section 2 a special kind of DAG was proposed to be a simplified model of natural deduction proofs. The considered method describes in a simple way a method of construction of the abstract proof graph for every reasoning that does not contain nested subreasonings. This demonstrates that the model is sound. It is natural now to give an argument that it is also complete, which means in this case that for every abstract proof graph there exists a reasoning whose structure is that graph. Now we show that this question has a positive answer.

For our purposes we consider a constructive subfamily of DAGs that includes graphs considered in Section 5. To study the general case of this subfamily we have to assume that $E \neq \mathfrak{R}(G)$ where $G=\langle V, E\rangle$ is an abstract proof graph with a distinguished set of arcs $\mathfrak{R}(G)$ (see p. 5) and $\mathfrak{R}(G)$ correspond to $E_{1}$ occurring in the instances of MIL problems. Since $\mathfrak{R}(G) \subsetneq E$, the constructed reasoning has to have some arcs other than reference arcs.

These non-reference arcs are represented in the reasoning with the help of a special kind of steps in the Mizar system. This special kind of steps in the general case has the shape:

$$
\begin{aligned}
& \text { consider variable be type such that } \\
& \text { label: statement by justification ; }
\end{aligned}
$$

These "consider" steps introduce new constants. Each step that uses such a constant is a head of a non-reference arc, the tail of which is in the corresponding "consider" step. For instance

$$
\begin{gathered}
\text { consider } \mathrm{x} \text { be set such that } \\
\mathrm{Ai}: \phi(\mathrm{x}) \text { by } \mathrm{Aj} ;
\end{gathered}
$$

is a tail of a non-reference arc with the head that contains the statement

$$
x=x
$$

in a step of the reasoning located after the step (6.2). Additionally, the verum in the Mizar language is stated as not contradiction.

Theorem 6.1. Let $G=\langle V, E\rangle$ be a $D A G$ and $E_{1}$ be a subset of $E$. Suppose that there exists a vertex which either as a head or as a tail belongs to arcs of $E_{1}$ only. Then there exists a correct Mizar reasoning that has the structure described by $G$ and the set of reference arcs equal to $E_{1}$.

Proof. Let $G, E_{1}$ fulfil assumptions and let $\mathfrak{t} \in V$ satisfy $\{\langle v, u\rangle \in E: v=\mathfrak{t} \vee u=\mathfrak{t}\} \subseteq E_{1}$. Let us fix a topological sorting $\tau \in T S(G)$. We introduce the following notation: $\mathcal{V} a r:=$ 
$\left\{v \in V: \underset{u \in V}{\exists}\langle v, u\rangle \in E \backslash E_{1}\right\}$ and

$$
\begin{aligned}
& \operatorname{Justification}(v):=\left\{\begin{aligned}
\text { by } \mathrm{A} \tau\left(u_{1}\right), \mathrm{A} \tau\left(u_{2}\right), \ldots, \mathrm{A} \tau\left(u_{k}\right) ; & \text { if } N_{\left\langle V, E_{1}\right\rangle}^{-}(v)=\left\{u_{1}, u_{2}, \ldots, u_{k}\right\} \wedge \\
& k>0, \\
; & \text { if } N_{\left\langle V, E_{1}\right\rangle}^{-}(v)=\emptyset,
\end{aligned}\right.
\end{aligned}
$$

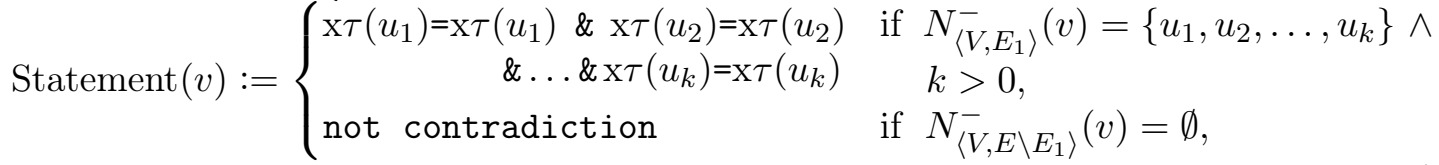

where $v \in V$. The reasoning step corresponding to a vertex $v$, denoted by $\operatorname{Step}(v)$, is defined as follows:

$$
\operatorname{Step}(v):= \begin{cases}\text { consider } \mathrm{x} \tau(v) \text { be set such that } & \text { if } v \in \mathcal{V} a r \\ \mathrm{~A} \tau(v): \mathrm{x} \tau(v)=\tau(v) \text { \& Statement }(v) \operatorname{Justification}(v) & \\ \mathrm{A} \tau(v): \operatorname{Statement}(v) \operatorname{Justification}(v) & \text { if } v \notin \mathcal{V} a r .\end{cases}
$$

If we prove that the reasoning

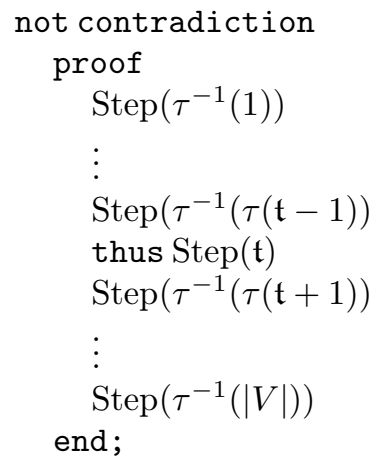

is correct and has expected properties, the proof will be completed. For illustration, an example of a reasoning that follows this pattern is presented in Fig. 7. Naturally, justifications in none of the steps are necessary for the Mizar system (an empty "semicolon" justification suffices to have it accepted by the checker), but a proof structure based on these justifications and statements has properties that are expected from the family of reference arcs and non-reference ones. Additionally, $\left\{u \in V:\langle u, v\rangle \in E \backslash E_{1}\right\} \subseteq \mathcal{V}$ ar for any $v \in V$. Moreover, none of variable identifiers are used before their introduction in reasoning. Analogously none of label identifiers are used before their introduction in reasoning. It is also easy to check that if Statement $(\mathfrak{t})=$ not contradiction then statement of the reasoning step thus $\operatorname{Step}(\mathfrak{t})$ is equal to the final goal, hence finally the proof is completed. 

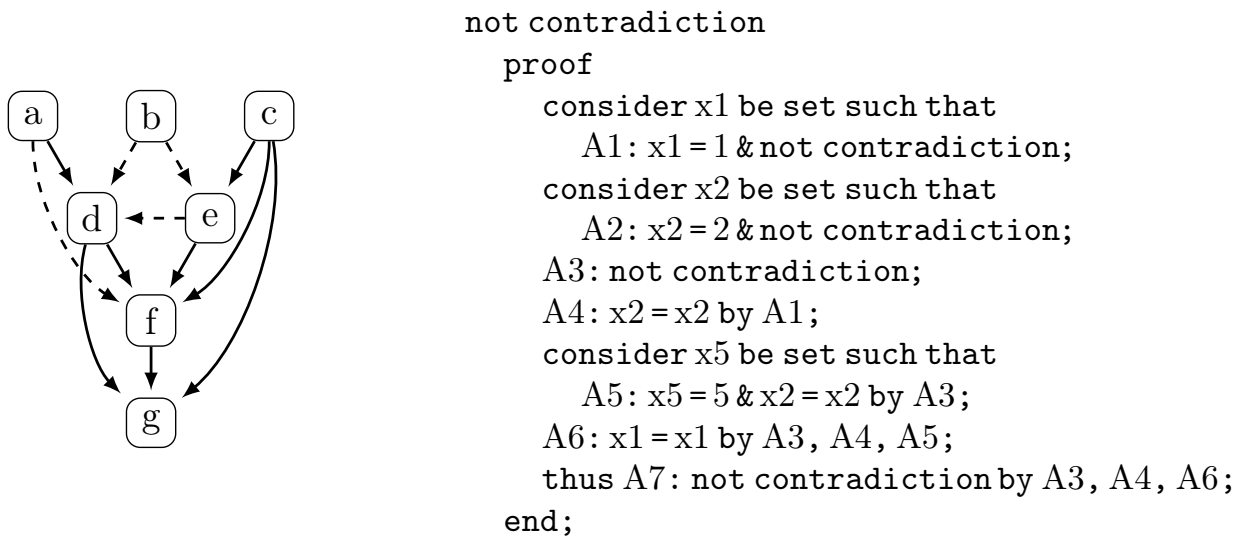

Figure 12: The Mizar proof script that illustrates the construction from the proof of Theorem 6.1. the solid arrows represent elements of $E_{1}$, dashed arrows represent elements of $E \backslash E_{1}, \mathfrak{t}=g, \mathcal{V} a r=\{a, b, e\}$ and $\tau(a)=1, \tau(b)=2, \ldots, \tau(g)=7$.

To be precise, the Mizar system will not accept many of the reasonings given by the construction in the proof. The reason is that the real system has a restriction on the number of references that can be used in the justification of a single step. In the official Mizar distribution this limit is 25, and this corresponds to the limit for the in-degree of every vertex in abstract proof graphs. This restriction is only used to bound the time of correctness verification of a single step and so far there was no need to increase this limit.

It is easy to see that the construction above can also serve to prove a parametrised version of Theorem 6.1 that takes into account this parameter.

Theorem 6.2. Let $G=\langle V, E\rangle$ be a $D A G$ with in-degree bounded by $k$ and $E_{1}$ be a subset of $E$. Suppose that there exists a vertex which either as a head or as a tail belongs to arcs of $E_{1}$ only. Then there exists a correct Mizar reasoning with the number of references that can be used in the justification bounded by $k$ and that has the structure described by $G$ as well as the set of reference arcs equal to $E_{1}$.

Note that both legibility improvement methods, 1st MIL and 2nd MIL, are NP-complete even with the limit 2 for the in-degree (see Th. 5.11).

\section{OTHER METHOdS OF IMPROVING PROOF READABILITY}

Various methods of improving readability of natural deduction proofs which use "then" $\tau$ steps have been considered in [29]. Now we sketch the complexity of optimization problems corresponding to the other proof readability criteria. It is not the main purpose of this paper, but it additionally illustrates the difficulty of improving the readability through the modification of the order of independent steps written in the proof script. These methods focus on the location of information used to justify a step and ones that are most similar to the methods considered in the current paper are:

(i) minimization of the largest distance between a premise and its use measured as the number of steps between these two steps in a linear representation of a proof script,

(ii) or minimization of the sum of all distances between every premise and its use. 
These methods of improving legibility of proofs can be formulated as the following two problems:

\section{3rd Method of Improving Legibility (3rd MIL):}

Instance: A DAG $G=\langle V, E\rangle$, a subset $E_{1}$ of $E$, and a positive integer $K \leq|V|$.

Question: Does there exist a topological sorting $\tau$ of $G$ for which

$$
\underset{\langle v, u\rangle \in E_{1}}{\forall} \tau(u)-\tau(v) \leq K ?
$$

\section{4th Method of Improving Legibility (4th MIL):}

Instance: A DAG $G=\langle V, E\rangle$, a subset $E_{1}$ of $E$, a positive integer $K \leq\left(\begin{array}{c}|V|+1 \\ 3\end{array}\right)$.

Question: Does there exist a topological sorting $\tau$ of $G$ for which

$$
\sum_{\langle v, u\rangle \in E_{1}} \tau(u)-\tau(v) \leq K ?
$$

Obviously the expression $\tau(u)-\tau(v)$ describes the distance between the pair of vertices $v, u$ and the arc $\langle v, u\rangle \in E_{1}$ describes the situation where the statement formulated in a reasoning step corresponding to the vertex $v$ is used as a premise in the justification of a step that corresponds to the vertex $u$.

Since every abstract proof graph $G=\langle V, E\rangle$ is constructible in the case $E=E_{1}$ (see Th. 6.1), it is easy to see that problems 3rd and 4th MIL generalize known NP-complete problems as Directed Bandwidth and Directed Optimal Linear Arrangement (see GT41, GT43 in [10]). Thus, we immediately conclude that these two problems belong to the class of NP-complete problems, too.

Another method of improving readability of the proofs considered in [29] concerns the number of steps that have to be marked with respective labels. As it was mentioned in Section 2, labels are used to pass on the information contained in a step to justify another one. Obviously, if some label is used only once in the following step and can be replaced by the "then" construction then this label can be removed. Therefore, it is possible to minimize the number of labels that are introduced in the proof script. Minimization of this parameter can be formulated as follows:

5th Method of Improving Legibility (5th MIL):

Instance: A DAG $G=\langle V, E\rangle$, a subset $E_{1}$ of $E$, and a positive integer $K \leq|V|$.

Question: Does there exist a topological sorting $\tau$ of $G$ for which

$$
\left\{v \in V: \underset{u \in V}{\exists}\langle v, u\rangle \in E_{1} \wedge \tau(u)-\tau(v)>1\right\}
$$

has size at most $K$ ?

We show in Theorem 7.1 below that the problem 5th MIL is NP-complete. However, the subfamily of abstract proof graphs considered in the proof of the theorem cannot be realized by proof scripts in MML due to an additional syntax restriction of Mizar. In the Mizar system a step of reasoning which introduces variables satisfying a statement which is 


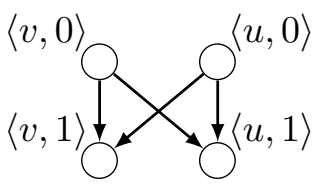

Figure 13: The subgraph of $G^{\prime}$ that corresponds to an edge $\{v, u\}$, which illustrates the construction from the proof of Theorem 7.1 .

used further in the script (see (6.2), the variable $\mathrm{x}$ satisfies $\phi(\mathrm{x})$ ) must be decorated with a label $(A i)$. This condition can be expressed in terms of graphs as follows: every vertex that is both a tail of some reference arc and a tail of some non-reference arc corresponds to a proof step with a label. As a result the minimalization must take the following form.

5th Method of Improving Legibility limited to the Mizar system (5th MIL MIZ $_{\text {): }}$

Instance: A DAG $G=\langle V, E\rangle$, a subset $E_{1}$ of $E$, and a positive integer $K \leq|V|$.

Question: Does there exist a topological sorting $\tau$ of $G$ for which

$$
\left\{v \in V: \underset{u \in V}{\exists}\langle v, u\rangle \in E_{1} \wedge\left(\tau(u)-\tau(v)>1 \vee \underset{w \in V}{\exists}\langle v, w\rangle \in E \backslash E_{1}\right)\right\}
$$

has size at most $K$ ?

It turns out that this limitation has a significant impact on the complexity of the task to minimise the number of labels. We show in Theorem 7.2 that the problem 5 th MIL $_{M I Z}$ is solvable in polynomial time. In consequence we get that it is possible to minimize the number of labels in the Mizar proof scripts effectively.

Theorem 7.1. 5th MIL in NP-complete.

Proof. We transform VC to 5th MIL. Let an undirected graph $G=\langle V, E\rangle$ and a positive integer $K \leq|V|$ be an instance of VC. We can assume that $G$ is without self-loops, i.e. without edges that connects a vertex to itself. We construct a directed graph $G^{\prime}=\left\langle V^{\prime}, E^{\prime}\right\rangle$ and a subset of $\operatorname{arcs} E_{1} \subset E^{\prime}$ such that there exists a vertex cover of $G$ with the size at most $K$ if and only if there exists a topological sorting $\tau \in T S\left(G^{\prime}\right)$ for which $|\mathfrak{L}(\tau)| \leq K$, where

$$
\mathfrak{L}(\tau):=\left\{v \in V^{\prime}: \underset{u \in V^{\prime}}{\exists}\langle v, u\rangle \in E_{1} \wedge \tau(u)-\tau(v)>1\right\} .
$$

Let $G^{\prime}, E_{1}$ be defined by

$$
\begin{aligned}
V^{\prime} & =V \times\{0,1\}, \\
E^{\prime} & =\{\langle\langle v, 0\rangle,\langle v, 1\rangle\rangle: v \in V\} \cup\{\langle\langle v, 0\rangle,\langle u, 1\rangle\rangle:\{v, u\} \in E\}, \\
E_{1} & =\{\langle\langle v, 0\rangle,\langle v, 1\rangle\rangle: v \in V\} .
\end{aligned}
$$

This translation can clearly be done in LOGSPACE. Notice that $G^{\prime}$ is acyclic since for each $v \in V$ the in-degree of vertices $\langle v, 0\rangle$ is 0 and out-degree of vertices $\langle v, 1\rangle$ is 0 . The main idea of the proofs uses the fact that since an edge $\{v, u\}$ belongs to $E$, at least one of $\langle v, 0\rangle$ or $\langle u, 0\rangle$ has to belong to $\mathfrak{L}(\tau)$, for every $\tau \in T S\left(G^{\prime}\right)$ (see Fig. 13). This is a consequence of the simple observation that at most one of two equalities $\tau(\langle v, 0\rangle)+1=\tau(\langle v, 1\rangle), \tau(\langle u, 0\rangle)+1=\tau(\langle u, 1\rangle)$ can hold for every $\tau \in T S\left(G^{\prime}\right)$.

Let $\mathcal{V}$ be a vertex cover of $G$. Let us consider a partition $\pi(\mathcal{V})$ of $G^{\prime}$ defined by

$$
\pi(\mathcal{V}):=\{\{\langle v, 0\rangle\}: v \in \mathcal{V}\} \cup\{\{\langle v, 1\rangle\}: v \in \mathcal{V}\} \cup\{\{\langle v, 0\rangle,\langle v, 1\rangle\}: v \in V \backslash \mathcal{V}\} .
$$


Notice that the only variables in $\mathcal{G}\left(G^{\prime}, \pi(\mathcal{V})\right)$ that have both in-degree and out-degree nonzero are the ones in $\{\{\langle v, 0\rangle,\langle v, 1\rangle\}: v \in V \backslash \mathcal{V}\}$. Moreover, the vertices of $\mathcal{G}\left(G^{\prime}, \pi(\mathcal{V})\right)$ in this set are not connected as $\mathcal{V}$ is a vertex cover. Then it is easy to check that $\mathcal{G}\left(G^{\prime}, \pi(\mathcal{V})\right)$ is acyclic and, in consequence, there exists $\tau \in T S\left(\mathcal{G}\left(G^{\prime}, \pi(\mathcal{V})\right)\right)$. Let $\sigma: V^{\prime} \rightarrow\left|\left\{1,2, \ldots, V^{\prime}\right\}\right|$ be a function given by $\sigma(\langle v, i\rangle)=j+\sum_{R \in \pi(\mathcal{V}): \tau(R)<\tau(P)}|R|$ where $P$ is the only one element of $\pi(\mathcal{V})$ that contains $\langle v, i\rangle ; j=1$ if $|P|=1$ and $j=i+1$ if $|P|=2$. It is also easy to check that $\sigma \in T S\left(G^{\prime}\right)$ and $\mathfrak{L}(\sigma) \subseteq\{\langle v, 0\rangle: v \in \mathcal{V}\}$, hence finally $|\mathfrak{L}(\sigma)| \leq|\mathcal{V}|$.

Let $\sigma$ be a topological sorting of $G^{\prime}$ and let $\mathcal{V}=\{v \in V:\langle v, 0\rangle \in \mathfrak{L}(\sigma)\}$. It is obvious that $|\mathcal{V}| \leq|\mathfrak{L}(\sigma)| \leq K$, hence we only need to show that $\mathcal{V}$ is a vertex cover of $G$. Suppose, contrary to our claim, that there exists an edge $\{v, u\} \in E$ such that $\{v, u\} \cap \mathcal{V}=\emptyset$. Then $\sigma(\langle v, 1\rangle)-\sigma(\langle v, 0\rangle) \leq 1$, since $\langle v, 0\rangle$ is the tail of $\langle\langle v, 0\rangle,\langle v, 1\rangle\rangle \in$ $E_{1}$. In the similar way, we claim that $\sigma(\langle u, 1\rangle)-\sigma(\langle u, 0\rangle) \leq 1$. Since $\sigma$ is a topological sorting of $G^{\prime}$ we have also that both $\sigma(\langle v, 0\rangle), \sigma(\langle u, 0\rangle)$ are less than both $\sigma(\langle v, 1\rangle), \sigma(\langle u, 1\rangle)$ (see Fig. 13). Additionally, these inequalities are between natural numbers, hence finally $\sigma(\langle v, 0\rangle)=\sigma(\langle u, 0\rangle), \sigma(\langle v, 1\rangle)=\sigma(\langle u, 1\rangle)$ and $v=u$, but this contradicts our assumption that $G$ is without self-loops.

Theorem 7.2. The 5 th $M I L_{M I Z}$ problem is solvable in polynomial time.

Proof. Let us consider an abstract proof graph $G=\langle V, E\rangle$ and an arbitrary subset $E_{1}$ of $E$. Note that every step corresponding to a vertex $v \in V$ for which one of the following properties holds

(i) $\left|\mathcal{N}_{\left\langle V, E_{1}\right\rangle}^{+}(v)\right|>1$ or

(ii) $\left|\mathcal{N}_{\left\langle V, E_{1}\right\rangle}^{+}(v)\right|=1$ and $\left|\mathcal{N}_{G}^{+}(v)\right|>1$

has to contain a label. Denote by $L_{1}$ the set of vertices that satisfy one of these conditions and let $\mathfrak{R}_{1}:=\left\{\langle v, u\rangle \in E_{1}: v \in L_{1}\right\}$. Naturally, at most one piece of information belonging to $\mathcal{N}_{\left\langle V, E_{1}\right\rangle}^{-}(v)$, can be passed on to justification of $v$ by the "then" construction, hence the other premises have to have labels. Let us construct a set $L_{2}$ that contains these premises. For each non-empty $\mathcal{N}_{\left\langle V, E_{1} \backslash \Re_{1}\right\rangle}^{-}(v)$ choose its subset $A_{v}$ of size $\left|\mathcal{N}_{\left\langle V, E_{1} \backslash \Re_{1}\right\rangle}^{-}(v)\right|-1$ and for empty $\mathcal{N}_{\left\langle V, E_{1} \backslash \Re_{1}\right\rangle}^{-}(v)$ let $A_{v}=\emptyset$. Let $L_{2} \subseteq V \backslash L_{1}$ be a union of $A_{v}$ for all $v \in V$. As $\mathcal{N}_{\left\langle V, E_{1} \backslash \Re_{1}\right\rangle}^{-}\left(v_{1}\right) \cap \mathcal{N}_{\left\langle V, E_{1} \backslash \Re_{1}\right\rangle}^{-}\left(v_{2}\right)=\emptyset$ for different $v_{1}, v_{2} \in V$, the choice of $A_{v}$ has no impact on the size of $L_{2}$.

With these considerations in mind we can conclude that every proof script has to have at least $\left|L_{1} \cup L_{2}\right|$ labels. Obviously sets $L_{1}, L_{2}$ can be determined in polynomial time. The proof is completed by showing that there exists a proof linearisation which uses exactly $\left|L_{1} \cup L_{2}\right|$ labels. For this purpose let us note that maximal length paths in the DAG $R:=\left\langle V, E_{1} \backslash\left(\mathfrak{R}_{1} \cup \mathfrak{R}_{2}\right)\right\rangle$ are node-disjoint and the graph $\mathcal{G}(R, \pi)$ is acyclic since only the last vertex in every maximal length path of $R$ can have the out-degree in $G$ greater than 1, where $\pi$ is a partition of $V$ defined by $\pi:=\{\mathcal{V}(P): P$ is a maximal length path in $R\}$, and $\mathfrak{R}_{2}=\left\{\langle v, u\rangle \in E_{1}: v \in L_{2}\right\}$. Obviously, vertices belonging to every element of $\pi$ can be ordered into a $\tau$-reasoning path and then setting these paths in order determined by an arbitrary topological sorting of $\mathcal{G}(R, \pi)$ we get a proof linearisation. 


\section{Conclusion}

In this paper we consider several problems arising in the course of improving readability of proof scripts. We concentrated on two methods of improving proof readability based on Behaghel's First Law. For these two methods we have found a common, unexplored problem AHP concerning graph partitions. This problem is similar to the problem of finding a partition of DAG into at most $K$ node-disjoint paths, which is solvable in polynomial time [4. With this in mind we can observe that the requirement of acyclicity imposed on the members of the partition is essential. Precisely this is the difference here.

Other three methods of improving proof readability are also solved but their solution was much simpler. These three methods generalize the previously described and solved graph problems. Additionally, many approximate solutions of these problems have been already found that can be adapted to the proof script case.

The next step in the process of improving proof readability should be finding the algorithms that approximate the problem AHP as well as the algorithms that approximate problems 1st MIL and 2nd MIL. It is also important to find heuristics that quickly give satisfactory approximate solutions to all five MIL problems. This should enable the possibility to make a decision which methods are more appropriate in particular cases.

\section{REFERENCES}

[1] K. K. Aggarwal, Y. Singh, and J.K. Chhabra. An integrated measure of software maintainability. In Reliability and Maintainability Symposium, pages 235-241, 2002.

[2] G. Bancerek and P. Carlson. A Synthesis of the Procedural and Declarative Styles of Interactive Theorem Proving. Logic in Computer Science, 8(1:30):1-26, 2012.

[3] O. Behaghel. Beziehungen zwischen Umfang und Reihenfolge von Satzgliedern. Indogermanische Forschungen, 25:110-142, 1909.

[4] N. Beldiceanu and X. Lorca. Necessary Condition for Path Partitioning Constraints. In P. V. Hentenryck and L. Wolsey, editors, 4th International Conference Integration of Artificial Intelligence and Operations Research Techniques in Constraint Programming for Combinatorial Optimization Problems, CPAIOR 200\%, volume 4510 of Lecture Notes in Computer Science, pages 141-154. Springer-Verlag, 2007.

[5] J. C. Blanchette. Redirecting Proofs by Contradiction. In Third International Workshop on Proof Exchange for Theorem Proving, PxTP 2013, volume 14 of EPiC Series, pages 11-26. EasyChair, 2013.

[6] M. Borowiecki and P. Mihók. Hereditary Properties of Graphs. Advances in Graph Theory. Vishwa International Publishers, 1991.

[7] P. Corbineau. A Declarative Language for the Coq Proof Assistant. In Proc. of the 2007 International Conference on Types for Proofs and Programs, pages 69-84, 2007.

[8] N. Cowan. Attention and Memory: An Integrated Framework. Oxford University Press, 1998.

[9] F. B. Fitch. Symbolic Logic: an Introduction. The Ronald Press Co., 1952.

[10] M. R. Garey and D. S. Johnson. Computers and Intractability: A Guide to the Theory of NPCompleteness. A Series of Books in the Mathematical Science. W. H. Freeman and Company, New York, 1979.

[11] E. Gibson. Linguistic complexity: locality of syntactic dependencies. Cognition, 68:1-6, 1998.

[12] M. Giero and F. Wiedijk. MMode, A Mizar Mode for the proof assistant Coq. Technical report, ICIS, Radboud Universiteit Nijmegen, 2004.

[13] G. Gonthier. A Computer-Checked Proof of the Four Colour Theorem. http://research.microsoft.com/en-us/um/people/gonthier/4colproof.pdf, 2005. [Online; accessed 22-May-2014].

[14] G. Gonthier. Formal Proof-The Four-Color Theorem. Notices of the AMS, 55(11):1382-1393, 2008.

[15] A. Grabowski, A. Korniłowicz, and A. Naumowicz. Mizar in a Nutshell. Journal of Formalized Reasoning, 3(2):153-245, 2010. 
[16] A. Grabowski and Ch. Schwarzweller. Improving Representation of Knowledge within the Mizar Library. Studies in Logic, Grammar and Rhetoric, 18(31):35-50, 2009.

[17] J. Harrison. A Mizar Mode for HOL. In Proc. of the 9th International Conference on Theorem Proving in Higher Order Logics, pages 203-220. Springer, 1996.

[18] M. Huth and M. Ryan. Logic in Computer Science, Modelling and Reasoning about Systems. Cambridge University Press, 2004.

[19] S. Jaśkowski. On the Rules of Supposition in Formal Logic. Studia Logica, 1934. Warszawa Reprinted in Polish Logic, ed. S.McCall, Clarendon Press, Oxford 1967.

[20] C. Kaliszyk and J. Urban. PRocH: Proof Reconstruction for HOL Light. In M. P. Bonacina, editor, 24th International Conference on Automated Deduction, CADE-24, volume 7898 of Lecture Notes in Computer Science, pages 267-274. Springer-Verlag, 2013.

[21] R. M. Karp. Reducibility Among Combinatorial Problems. Plenum Press New York, Complexity of Computer Computations:85-103, 1972.

[22] A. Korniłowicz. Tentative Experiments with Ellipsis in Mizar. In J. Jeuring, J. A. Campbell, J. Carette, Gabriel G. Dos Reis, P. Sojka, M. Wenzel, and V. Sorge, editors, Intelligent Computer Mathematics 11th International Conference, volume 7362 of Lecture Notes in Artificial Intelligence, pages 453-457. Springer-Verlag, 2012.

[23] A. Kornilowicz. On Rewriting Rules in Mizar. Journal of Automated Reasoning, 50(2):203-201, 2013.

[24] E. Kusak, W. Leończuk, and M. Muzalewski. Abelian Groups, Fields and Vector Spaces. Formalized Mathematics, 1(2):335-341, 1990.

[25] R. Levy. Expectation-based syntactic comprehension. Cognition, 106(2008):1126-1177, 2007.

[26] W. Marciszewski. A Jaśkowski-Style System of Computer-Assisted Reasoning. Philosophical Logic in Poland, Kluwer, 1993.

[27] A. Naumowicz. Interfacing external CA systems for Grobner bases computation in Mizar proof checking. International Journal of Computer Mathematics, 87(1):1-11, January 2010.

[28] A. Naumowicz and A. Korniłowicz. A Brief Overview of Mizar. In Proc. of International Conference on Theorem Proving in Higher Order Logics, TPHOLs'09, volume 5674 of Lecture Notes in Computer Science, pages 67-72. Springer-Verlag, 2009.

[29] K. Pąk. The Algorithms for Improving and Reorganizing Natural Deduction Proofs. Studies in Logic, Grammar and Rhetoric, 22(35):95-112, 2010.

[30] K. Pąk. Methods of Lemma Extraction in Natural Deduction Proofs. Journal of Automated Reasoning, 50(2):217-228, 2013.

[31] S. P. Rahul and George C. Necula. Proof Optimization Using Lemma Extraction. UCB/CSD-01-1143, Computer Science Division (EECS), University of California, 2001.

[32] P. Rudnicki. Obvious Inferences. Journal of Automated Reasoning, 3(4):383-393, 1987.

[33] S. J. Smolka and J. C. Blanchette. Robust, Semi-Intelligible Isabelle Proofs from ATP Proofs. In Third International Workshop on Proof Exchange for Theorem Proving, PxTP 2013, volume 14 of EPiC Series, pages 117-132. EasyChair, 2013.

[34] E. Snapper. The three crises in mathematics: Logicism, intuitionism and formalism. Mathematics Magazine, 52(4):207-2016, 1979.

[35] D. Syme. Three Tactic Theorem Proving. In Theorem Proving in Higher Order Logics, volume 1690 of Lecture Notes in Computer Science, pages 203-220. Springer-Verlag, 1999.

[36] W. A. Trybulec. Groups. Formalized Mathematics, 1(5):821-827, 1990.

[37] W. A. Trybulec. Vectors in Real Linear Space. Formalized Mathematics, 1(2):291-296, 1990.

[38] J. Urban. XML-izing Mizar: Making Semantic Processing and Presentation of MML Easy. In M. P. Bonacina, editor, 4th International Conference Mathematical Knowledge Management 2005, MKM'05, volume 3863 of Lecture Notes in Computer Science, pages 346-360. Springer-Verlag, 2005.

[39] J. Urban. MizarMode - An Integrated Proof Assistance Tool for the Mizar Way of Formalizing Mathematics. Journal of Applied Logic, 4(4):414-427, 2006.

[40] J. Urban, P. Rudnicki, and G. Sutcliffe. ATP and Presentation Service for Mizar Formalizations. Journal of Automated Reasoning, 50(2):229-241, 2013.

[41] M. Wenzel. Isabelle/jEdit. University of Cambridge, 2013.

[42] M. Wenzel. The Isabelle/Isar Reference Manual. University of Cambridge, 2013.

[43] F. Wiedijk. Mizar Light for HOL Light. Proc. of the 14th International Conference on Theorem Proving in Higher Order Logics, pages 378-394, 2001. 
[44] V. Zammit. On the Readability of Machine Checkable Formal Proofs. PhD thesis, The University of Kent at Canterbury, March 1999.

This work is licensed under the Creative Commons Attribution-NoDerivs License. To view a copy of this license, visit http://creativecommons.org/licenses/by-nd/2.0/ or send a letter to Creative Commons, 171 Second St, Suite 300, San Francisco, CA 94105, USA, or Eisenacher Strasse 2, 10777 Berlin, Germany 